\title{
Gerinnungsprobleme in der Intensivmedizin
}

Ludwig Ney, Michael Spannagl

\section{Störungen des Gerinnungssystems und konsekutive hämorrhagische oder thromboembolische Komplikationen sind in der Intensivmedizin ebenso häufige wie ernsthafte Probleme. Ihre frühzeitige Erkennung, korrekte diagnostische Ein- ordnung und gezielte Behandlung sind entscheidende Faktoren für das Outcome.}

\section{Einleitung}

Sowohl eine eingeschränkte Hämostasekapazität mit den daraus resultierenden Blutungskomplikationen als auch Thromboembolien mit ihren makro- und mikrozirkulatorischen Auswirkungen können aus intensivmedizinischer Sicht als Komponenten der Multiorgandysfunktion bzw. des Multiorganversagens interpretiert werden. Sie können - wie andere Einschränkungen von Organfunktionen (z. B. ARDS, akutes Nierenversagen) jeweils Auslöser, pathophysiologischer Co-Faktor oder Folge des kritischen Krankheitszustands sein. Nicht selten treten sie kombiniert und in verschiedenen Stadien des Krankheitsgeschehens wiederholt auf.

Dieser Artikel behandelt Gerinnungsstörungen im Sinne einer reduzierten hämostaseologischen Kapazität im intensivmedizinischen Bereich. Schwerpunkte sollen das Problem der mittlerweile häufig vorbestehenden medikamentösen Antikoagulation bzw. Thrombozytenhemmung sein und deren Auswirkung auf Diagnostik und Therapie, außerdem die Besonderheiten der Gerinnungsaktivierung bei akuter Blutung und bei septischen Krankheitsbildern, der Umgang mit seltenen Gerinnungsstörungen sowie die Bedeutung thrombozytärer Funktionsstörungen in der Intensivmedizin. Gerinnungsstörungen aufgrund von Leber- und Nierenkrankheiten sind nicht Gegenstand dieses Artikels.

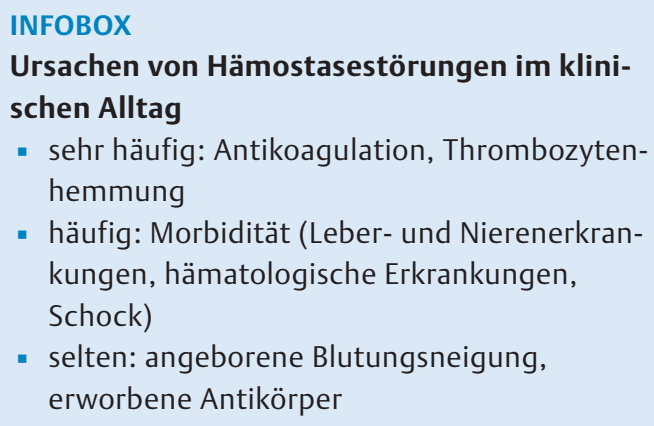

- sehr häufig: Antikoagulation, Thrombozytenhemmung

- häufig: Morbidität (Leber- und Nierenerkrankungen, hämatologische Erkrankungen, Schock)

- selten: angeborene Blutungsneigung, erworbene Antikörper

\section{Blutstillung vs. Antikoagulation}

Intensivpatienten sind häufig durch Verletzungen oder innere Läsionen (z.B. Ulzera, Aneurysmen), Operationen und Interventionen akut blutungsgefährdet. Gleichzeitig oder im schnellen Wechsel bestehen aber auch erhebliche prädisponentielle und expositionelle Risikofaktoren für thromboembolische Komplikationen, sodass eigentlich eine Indikation für eine zumindest prophylaktische, ggf. auch therapeutische Antikoagulation gegeben ist. Hierbei sollten wir nicht aus den Augen verlieren, dass fast alle Thromboserisiken, die zur Indikation einer prophylaktischen Antikoagulation führen, potenzieller Natur sind. Umgekehrt sind die Folgen einer manifesten Blutung ein höchst konkretes Risiko.

Bei der sich daraus ergebenden Risikobewertung müssen wir uns zudem den Ablauf hämostaseologischer Prozesse vor Augen halten: Die primäre Hämostase verläuft ungehemmt schnell (Minuten bis Stunden) und endgültig. Wenn ein fester Thrombus an einer Läsion etabliert ist, kann er durch die üblichen Methoden der antikoagulatorischen und antithrombozytären Therapie nicht mehr aufgelöst werden, sondern nur noch durch körpereigene oder extern zugeführte Fibrinolytika oder aber mechanische Interventionen. Umgekehrt basieren die meisten Indikationen zur Gerinnungshemmung auf einem zumindest etwas längerfristigen (Tage bis Wochen) oder statistischen Thrombembolierisiko.

Aufgrund dieser Überlegungen hinsichtlich Risiko und relevanter Zeiträume ergibt sich, dass man - soweit irgend möglich - postoperativ oder in Blutungssituationen zunächst die primäre Hämostase ermöglichen sollte und erst danach mit einer therapeutischen oder prophylaktischen Antikoagulation oder antithrombozytären Therapie beginnen sollte. Ausnahmen sind z. B. frische Koronarstents sowie bestimmte gefäßchirurgische oder interventionelle rekanalisierende Eingriffe, 


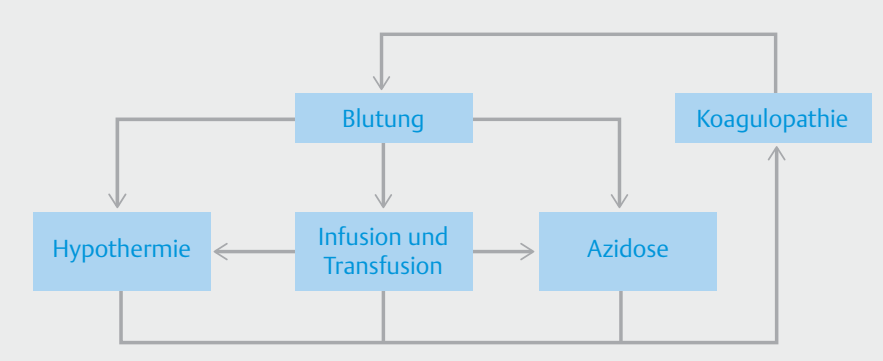

- Abb. 1 Die klassische „Lethal Triade“ (Hypothermie, Azidose, Koagulopathie) als Folge einer akuten Blutung ist hier um die Infusions- und Transfusionstherapie zum „tödlichen Quartett“ ergänzt.

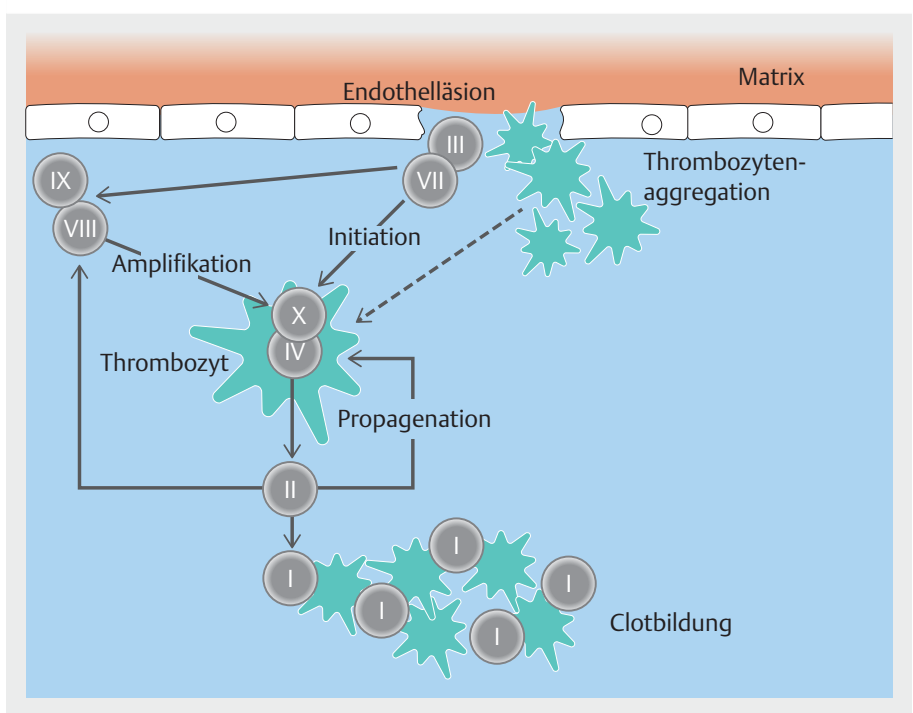

- Abb. 2 Integriertes Modell der Gerinnung.

bei denen jede Antikoagulationspause das Ergebnis und damit evtl. den Erhalt einer Extremität - bedroht.

Merke

Unter aktiver Blutung keine Gabe von Inhibitoren der Gerinnung oder der Thrombozytenfunktion - es sei denn, es besteht eine absolute und zeitdringliche Indikation.

\section{Ungestillte Blutung: Ursache und Folge von Gerinnungsstörungen}

Das wichtigste Zeichen einer drohenden (oder bereits eingetretenen) Grenze der hämostaseologischen Kapazität ist im klinischen Alltag die (noch) nicht gestillte Blutung. Eindrücklich zeigt das Bild der „Lethal Triade“ die Problemkonstellation ( $\triangleright$ Abb. 1): Die manifeste Blutung kann weder mit chirurgischen Maßnahmen noch mit einer hämostaseologischen Substitution oder durch Versuche einer kardiozirkulatorischen Stabilisierung alleine, sondern nur mit einer Kombination aller 3 Komponenten gestillt werden. Hierbei führt die Blu- tung zu einer komplexen Gerinnungsstörung, die neben einem Substratmangel für die Thrombusbildung (Fibrinogen, Thrombozyten, Erythrozyten) auch zu einer Erschöpfung weiterer pro- und antikoagulatorisch wirksamen Faktoren führt und daher einer differenzierten Therapie bedarf.

\section{Auslösen der Blutgerinnung}

Die klassische Sichtweise eines getrennten zellulären und plasmatischen Gerinnungssystems, Letzteres noch geteilt in einen ex- und intrinsischen Weg, wurde zu einem integrierten Modell der Hämostase vereint ( $\triangleright$ Abb. 2) [1]:

- In der Initiationsphase bewirkt die Endothelläsion durch die Freisetzung von Gewebethromboplastin („tissue factor“, Faktor III) zusammen mit Faktor VII einerseits die Aktivierung der Faktoren $\mathrm{X}$ und $\mathrm{V}$ und verursacht andererseits durch die Freilegung der subendothelialen Matrix eine Thrombozytenaggregation.

- Die Faktoren X und V sind dabei auf der Thrombozytenoberfläche gebunden und stehen daher an der Läsion lokal in hoher Konzentration zur Verfügung, was zu der wünschenswerten Ortsselektivität der Gerinnung führt. Gleichzeitig verstärken die frühzeitig (mit-) aktivierten Faktoren IX und VIII die Gerinnungsaktivierung. Daher wird diese Phase als Amplifikation bezeichnet, die unmittelbar zur Thrombinbildung (Faktor II) führt. Durch Thrombin wird die Thrombusbildung ausgelöst, da es sowohl eine Fibrinbildung (Faktor I) als auch eine massive Thrombozytenaktivierung hervorruft.

- Die Thrombinaktivierung verstärkt sich schlagartig (Thrombin-Burst) über autokrine (zwischen den Thrombozyten) und parakrine (über die Faktoren XII, IX, VIII) Rückkopplungswege. Man spricht von der Propagenationsphase. Dadurch bildet sich das Netzwerk aus Fibrin und Thrombozyten unter Einbeziehung von Erythrozyten, das konsekutiv durch Faktor XIII stabilisiert wird.

\section{Diagnostik und Überwachung von Gerinnungsstörungen}

\section{Klinische Beurteilung}

Die klinische Beobachtung einer akuten Blutung muss sofort auch an eine bereits manifeste oder drohende Gerinnungsstörung denken lassen. Ebenso wie Erythrozyten verloren gehen bzw. verdünnt werden, kommt es zu einem Abfall der hämostatischen Faktoren. Ausgerechnet Fibrinogen und Thrombozyten, die Substrate für den hämostatischen Thrombus (s.u.), fallen bei fortschreitender Blutung als erste Einzelfaktoren unter die kritische Grenze ihrer Wirksamkeit. Die mangelnde Thrombusbildung führt dann zu Blutungen auch aus kleinen Läsionen wie Stichkanälen, oberflächlichen 
Verletzungen und zu Spontanblutungen aus Schleimhäuten auch außerhalb der Zone der eigentlichen Verletzung oder des OP-Situs. Daher spricht man dann von diffusen Blutungen.

\section{Merke}

Das Auftreten diffuser Blutungen ist das wichtigste Leitsymptom einer akuten Koagulopathie.

\section{Prädiktion und Risikobeurteilung}

\section{Grenzen der verfügbaren Diagnostik}

Die Antizipation möglicher Komplikationen ist eine zentrale Kompetenz der Intensivmedizin, hängt von ihr doch wesentlich die Indikation einer Intensivüberwachung, die Bereitstellung von Ressourcen sowie Zeitpunkt und Wirksamkeit einer potenziell erforderlichen Intervention ab. Dies gilt insbesondere für die Gerinnungstherapie, die wesentlich auf einer ggf. sehr teuren, aber bisweilen frühzeitig und hochdosiert erforderlichen Substitutionstherapie beruht.

Die anfängliche Einschätzung und weitere Überwachung der hämostatischen Funktion basiert auf der klinischen Beobachtung, einer strukturierten Anamnese und der Erfassung bestimmter Begleitfaktoren sowie auf hämostaseologischen In-vitro-Tests als der klassischen Gerinnungsdiagnostik.

Eine intakte Gerinnungskapazität ist die beste Prophylaxe einer akuten Blutung und die wichtigste Voraussetzung für ihre Beherrschung. Stärker als endogene Gerinnungsstörungen stellt uns die steigende Zahl dauerhaft therapeutisch antikoagulierter oder thrombozytenaggregationsgehemmter Patienten vor klinische Herausforderungen - nicht nur wegen der damit verbundenen eingeschränkten hämostaseologischen Kapazität, sondern insbesondere wegen eines erforderlichen diagnostischen Paradigmenwechsels.

Quick und PTT als schnell und überall verfügbare Globaltests zeigten bisher das Ausmaß sowohl einer primär endogenen (also krankheitsbedingten) als auch exogenen (therapeutischen) plasmatischen Antikoagulation. Letztere wurde über Jahrzehnte ambulant meist mit Vitamin-K-Antagonisten und stationär mit Heparin durchgeführt. Seit der Einführung der niedermolekularen Heparine und mehr noch der direkten oralen Antikoagulanzien (DOAK) büßen diese Globaltests ihre diagnostische Aussagekraft zusehends ein, da sich die antikoagulatorische Wirkung dieser Substanzen allenfalls bei erheblicher Überdosierung zuverlässig in den Globaltest niederschlägt-dann aber oft bereits einhergehend mit einer manifesten Blutung. Ein Ersatz der Globaltests mit ausreichender Sensitivität bezüglich der Wirkung bzw. des Risikos aller gebräuchlicher Antikoagulanzien ist derzeit nicht in Sicht.
Zudem ist eine Prüfung der Thrombozytenfunktion im Routinelabor nicht verfügbar, sodass nur ihre Zahl im Blutbild gemessen werden kann. Die Ergebnisse moderner funktioneller Messsysteme der Thrombozytenfunktion wie PFA-100 oder Multiplate wiederum sind nur bei normaler Erythrozyten- und Thrombozytenzahl aussagekräftig.

\section{Prädiktive Modelle in Akutsituationen}

Um also frühzeitig, idealerweise auch in der Akutsituation noch präventiv, auf eine relevante hämostaseologische Einschränkung und die resultierende Blutungsgefahr aufmerksam zu werden, brauchen wir prädiktive Modelle, die auf einer strukturierten Anamnese- und Befunderhebung beruhen.

Bei schwer verletzten Patienten steht akut weniger die Frage im Vordergrund, ob es überhaupt zu Blutungen kommt, sondern vielmehr die Überlegung, wie diese beherrscht werden können. Neben den erforderlichen chirurgischen Maßnahmen steht in diesem Zusammenhang die Transfusions- und Substitutionstherapie im Zentrum. Bereits in den ersten Minuten muss festgelegt werden, welche Zugänge der Patient braucht, ob eine Transfusions- und Substitutionstherapie bereits vor dem Erreichen diagnostischer Kenngrößen eingeleitet werden soll und welche Blutprodukte bereitgestellt werden müssen.

Für die rasche Beantwortung dieser Fragen sind physiologische Einzelwerte ungeeignet, da sie auch anderen Faktoren unterliegen und daher wenig spezifisch sind (z.B. Tachykardie durch Blutverlust und/oder durch Schmerzen) oder da sie durch Kompensationsmechanismen unzureichend oder erst verzögert das Ausmaß des Traumas widerspiegeln (z. B. arterielle Hypotonie) und daher wenig sensitiv sind. Deshalb wurden verschiedene Scores entwickelt, die aus einer Kombination klinischer und hämatologischer Parameter den Transfusionsbedarf vorhersagen sollen ( $\downarrow$ Tab. 1). In einem direkten Vergleich erwies sich der besonders einfach anzuwendende $A B C$-Score als überlegen, allerdings mit vielen falsch positiven Ergebnissen [2].

In der internistischen Akutmedizin gibt es erkrankungsspezifische Scores für Patienten mit oberer gastrointestinaler Blutung (Glasgow-Blatchford Bleeding Score) [6] oder mit akutem Koronarsyndrom (CRUSADE Score) [7]. Hierbei werden neben Alter und Geschlecht Kreislaufparameter, Laborwerte und Begleiterkrankungen abgefragt.

Der Glasgow-Blatchford Bleeding Score für Patienten mit oberer gastrointestinaler Blutung wird zum Aufnahmezeitpunkt erhoben und erwies sich als prädiktiv bezüglich des Interventionsbedarfs, des Transfusionsbedarfs und der Krankenhausaufenthaltsdauer. Hierfür werden 
- Tab. 1 Scores zur Vorhersage einer erforderlichen Massivtransfusion und die dabei verwendeten Parameter. Bei ABC werden die abgefragten Punkte addiert, bei einer Summe $\geq 2$ ist eine Massivtransfusion wahrscheinlich erforderlich. TASH und McLaughlin gewichten die abgefragten Parameter mit definierten Algorithmen. Der TASH-Score wurde anhand des Traumaregisters der Deutschen Gesellschaft für Unfallchirurgie entwickelt. McLaughlin et al. entwickelten ihren Score ausschließlich anhand kriegsverletzter Patienten.

\begin{tabular}{|c|c|c|c|}
\hline Parameter & $A B C[3]$ & TASH [4] & McLaughlin [5] \\
\hline penetrierende Verletzung & . & & \\
\hline Beckenfraktur & & . & \\
\hline Femurfraktur & & . & \\
\hline Tachykardie & . & . & . \\
\hline Hypotonie & . & . & . \\
\hline thorakale oder abdominelle Blutung in der primären Sonografie (FAST) & . & . & \\
\hline Hb-Konzentration & & . & . \\
\hline Base Excess & & - & \\
\hline $\mathrm{pH}$ & & & . \\
\hline Geschlecht & & . & \\
\hline
\end{tabular}

Parameter graduell (Harnstoff-Stickstoff im Blut, Hämoglobinkonzentration und systolischer Blutdruck) oder kategorisch (Tachykardie, Meläna, Synkope, Lebererkrankungen, Herzinsuffizienz) in Punktwerte übersetzt, deren Summe zwischen 0 und 23 liegt. Hinsichtlich der Vorhersage des Therapiebedarfs ist er anderen Scores (Rockall, AIMS65) überlegen.

Der CRUSADE Score soll das periinterventiontionelle Blutungsrisiko ursprünglich bei Nicht-ST-Hebungsinfarkten vorhersagen. Wie beim Glasgow-Blatchford Bleeding Score werden Parameter graduell (Hämatokrit, Kreatinin-Clearance, Herzfrequenz, systolischer Blutdruck) oder kategorisch (Geschlecht, dekompensierte Herzinsuffizienz, Gefäßerkrankung, Diabetes) in eine Punktsumme zwischen 0 und 100 übersetzt. Das Blutungsrisiko beträgt bei weniger als 20 Punkten $3 \%$, bei über 50 Punkten bis 19\%. Der Score wurde multizentrisch aus Daten von über 70.000 Patienten entwickelt und erreicht in der "receiver operating characteristic“ eine „area under the curve“ (AUC) von über 0,7.

Therapeutische Antikoagulation und Blutungsrisiko bei chronischen Krankheiten

Die Zahl von Patienten, die akut oder noch häufiger chronisch antikoaguliert sind, nimmt derzeit rapide zu. Ursache hierfür ist nicht allein die Zunahme an spezifischen Krankheiten der älter werdenden Gesellschaft, sondern auch ein höherer Stellenwert der antikoagulatorischen Therapie in der Primär- und Sekundärprophylaxe sowie ein verbessertes Sicherheitsprofil der direkten oralen Antikoagulanzien (DOAK). Häufige Indikationen für eine therapeutische Antikoagulation (plasmatisch oder thrombozytär) sind in > Tab. 2 zusammengefasst.
Von besonderer Bedeutung ist die antithrombozytäre Therapie bei der KHK. Besonders seit der Einführung der „drug eluting stents“ (DES) hat der Anteil von Patienten mit dualer Thrombozytenaggregationshemmung deutlich zugenommen. Der Therapie dient fast durchweg eine Kombination aus ASS und einem $\mathrm{P}_{2} \mathrm{Y}_{12}$-Inhibitor.

Die aktuell von den amerikanischen Kardiologie-Leitlinien empfohlenen Mindestzeiten einer dualen Thrombozytenaggregationshemmung zeigt $>$ Tab. $\mathbf{3}$.

Bei der letzten Aktualisierung der Leitlinien wurde auch das Vorgehen bei elektiven nicht-herzchirurgischen Operationen modifiziert, da die neueren Stents ein geringeres Risiko einer In-Stent-Thrombose aufweisen. Daher können ggf. die in > Tab. 3 angegebenen Zeiten unterschritten werden:

- Bei Patienten unter dualer Thrombozytenaggregationshemmung nach DES-Implantation sind elektive Operationen in den ersten 3 Monaten nach PCI weiterhin klar kontraindiziert.

- Im Zeitraum von 3-6 Monaten nach dem Eingriff ist eine individuelle Risikoabwägung erforderlich (IIb).

- Nach 6 Monaten ist eine Operation nach Absetzen des $\mathrm{P}_{2} \mathrm{Y}_{12}$-Inhibitors vertretbar (I).

Klar ist: Eine Verkürzung der dualen Plättchenhemmung steigert potenziell das kardiologische Risiko und setzt daher eine interdisziplinäre Entscheidungsfindung (Anästhesie, Chirurgie, Innere Medizin) und die aufgeklärte Zustimmung des Patienten voraus.

Um das Blutungsrisiko individuell abschätzen zu können, wurden für Patienten mit Vorhofflimmern unter Antikoagulation mehrere Scores entwickelt. Eine Orientierung an diesen Scores kann die Anamnese- und 
- Tab. 2 Häufige Indikationen zur Gerinnungshemmung und die hierfür hauptsächlich eingesetzte Therapieform. Strenge Indikation bedeutet, dass auch kurze Pausen (z. B. perioperativ) der Dauermedikation möglichst vermieden oder durch Alternativpräparate überbrückt werden müssen (DES: „drug eluting stent“).

\begin{tabular}{|c|c|c|c|}
\hline Indikation & $\begin{array}{l}\text { Thrombozytenaggregations- } \\
\text { hemmung }\end{array}$ & Gerinnungshemmung & $\begin{array}{l}\text { Strenge Indikation } \\
\text { (Minimum) }\end{array}$ \\
\hline Vorhofflimmern & & • & \\
\hline KHK, akutes Koronarsyndrom & - & & $\begin{array}{l}\text { bei DES: } 6 \text { - } 12 \text { Monate duale } \\
\text { Thrombozytenhemmung }\end{array}$ \\
\hline periphere arterielle Verschlusskrankheit & • & - & \\
\hline zerebrovaskuläre Erkrankung & • & & \\
\hline venöse Thromboembolie & & - & $6-8$ Wochen \\
\hline mechanischer Herzklappenersatz & & - & lebenslang \\
\hline
\end{tabular}

Tab. 3 Mindestzeiten der dualen Thrombozytenaggregationshemmung bei KHK [8]. Der Empfehlungsgrad ist jeweils in Klammern angegeben. Eine Therapiedauer über den angegebenen Zeitraum hinaus ist jeweils eine Ilb-Empfehlung bei fehlender Blutungsneigung (PCI: „percutaneous coronary intervention“; BMS: „bare metal stent“; DES: „drug eluting stent“; CABG: „coronary artery bypass graft“).

\begin{tabular}{|l|l|l|l|l|}
\hline & Konservativ & Lyse ohne PCI & PCI mit BMS & PCI mit DES \\
\hline stabile Erkrankung, elektive Versorgung & kein Nutzen & - & $\geq 1$ Monat (I) & $\geq 6 \mathrm{Monate}(\mathrm{I})$ \\
\hline akutes Koronarsyndrom, Notfallversorgung & & $\geq 12$ Monate (IIb) \\
\hline
\end{tabular}

Befunderhebung strukturieren und für die klinische Interpretation gewichten. T Tab. 4 zeigt die Scores, die in der aktuellen europäischen Leitlinie zur Behandlung von Vorhofflimmern erwähnt werden [9]. HASBLED wurde dabei an Patienten unter Vitamin-K-Antagonisten (z. B. Phenprocoumon) entwickelt, ORBIT und $A B C$ an Patienten unter Vitamin-K-Antagonisten oder DOAK.

\section{In-vitro-Diagnostik}

\section{Hämostaseologische Globaltests}

Im Zentrum der In-vitro-Diagnostik stehen neben dem kleinen Blutbild (Hämatokrit und Thrombozytenzahl) die Prothrombinzeit (PT, Synonym: Thromboplastinzeit, Quick-Wert) bzw. INR und die partielle aktivierte Thromboplastinzeit (aPTT). Die Vorteile dieser Parameter sind ihre meist durchgehende Verfügbarkeit in nahezu jedem klinisch-chemischen Labor und ihr günstiger Preis, der sie auch zum Überwachung des Verlaufs einer Gerinnungsstörung oder Therapie prädestinieren. Gleichzeitig aber gestatten sie als Globaltests noch keine klare Identifizierung der Ursache einer Gerinnungsstörung ( $\triangleright$ Tab.5). Zur Ursachensuche setzt man in der Labordiagnostik überwiegend Konzentrations- oder Aktivitätsmessungen von Einzelfaktoren ein, die aber aufwendig und nicht überall und rasch verfügbar sind.
- Tab. 4 Vergleich der bei verschiedenen Blutungsscores erfassten Parameter (GDF-15: „growth differentiation factor 15“, erhöht bei Nierenfunktionsstörungen. Alternativ zur Verwendung von GDF-15 gibt es auch Berechnungsmodelle anhand von Cystatin C oder der glomerulären Filtrationsrate).

\begin{tabular}{|c|c|c|c|}
\hline Parameter & HAS-BLED [10] & ORBIT [11] & $\mathrm{ABC}[12]$ \\
\hline Lebererkrankung & . & & \\
\hline Niereninsuffizienz & . & . & \\
\hline Alkoholabusus & . & & \\
\hline Alter & $>65$. & $\geq 75$. & - \\
\hline Schlaganfall & · & & \\
\hline instabile INR & . & & \\
\hline Blutungsanamnese & . & . & . \\
\hline $\begin{array}{l}\text { Thrombozyten- } \\
\text { aggregationshemmer }\end{array}$ & . & . & \\
\hline $\begin{array}{l}\text { schlecht eingestellte } \\
\text { Hypertonie }\end{array}$ & . & & \\
\hline $\begin{array}{l}\text { Anämie/ } \\
\text { Hb-Konzentration }\end{array}$ & & . & . \\
\hline Troponin T & & & · \\
\hline GDF-15 & & & . \\
\hline c-Index [12] & 0,61 & 0,65 & 0,71 \\
\hline
\end{tabular}


- Tab. 5 Verlängerung von Prothrombinzeit (PT) bzw. INR und aPTT sowie typische Ursachen (DIC: disseminierte intravasale Gerinnung, NMH: niedermolekulares Heparin, UFH: unfraktioniertes Heparin, VKA: Vitamin-K-Antagonisten, vWS: von-Willebrand-Syndrom).

\begin{tabular}{|c|c|c|}
\hline PT/INR & aPTT & Typische Ursachen \\
\hline \multirow[t]{5}{*}{$\uparrow$} & \multirow[t]{5}{*}{$\uparrow$} & $\begin{array}{l}\text { - Störung der „Endstrecke“ (Faktoren X, V, II, I) } \\
\text { einschließlich hoch-/überdosierter Antikoagu- } \\
\text { lation mit Xa- (Rivaroxaban, Apixaban, } \\
\text { Edoxaban, NMH) und Thrombininhibitoren } \\
\text { (Dabigatran, Agatroban) }\end{array}$ \\
\hline & & - Verbrauchskoagulopathie/DIC \\
\hline & & $\begin{array}{l}\text { - Überdosierung einer Antikoagulation bzw. } \\
\text { kombinierte Wirkung von VKA+UFH }\end{array}$ \\
\hline & & - Vergiftungen (Rattengift) \\
\hline & & - hepatische Synthesestörungen \\
\hline \multirow[t]{4}{*}{$\uparrow$} & \multirow[t]{4}{*}{$\leftrightarrow$} & $\begin{array}{l}\text { - Störungen des extrinsischen Wegs, } \\
\text { insbesondere Faktor VII }\end{array}$ \\
\hline & & $\begin{array}{l}\text { - Verbrauchskoagulopathie, disseminierte } \\
\text { intravasale Gerinnung }\end{array}$ \\
\hline & & - Antikoagulation mit VKA \\
\hline & & - hepatische Synthesestörungen \\
\hline \multirow[t]{2}{*}{$\leftrightarrow$} & \multirow[t]{2}{*}{$\uparrow$} & $\begin{array}{l}\text { - Störungen des intrinsischen Wegs (Faktoren } \\
\text { VIII, IX, XI, XII) einschließlich angeborener oder } \\
\text { erworbener Hämophilien }\end{array}$ \\
\hline & & $\begin{array}{l}\text { - angeborenes oder erworbenes vWS (variabler } \\
\text { Einfluss auf die aPTT) }\end{array}$ \\
\hline
\end{tabular}

Messung der Wirkung direkter oraler Antikoagulanzien

Die klinisch an Bedeutung gewinnenden DOAK erlauben keine einfache Therapiesteuerung anhand der Globaltests wie die konventionellen Antikoagulanzien Heparin (mit PTT) oder Vitamin-K-Antagonisten (mit Quick/INR). DOAK beeinflussen die Wirkung der aktivierten Faktoren X und II (Thrombin). Daher beeinflussen direkte Hemmer dieser Enzyme Quick und PTT in Abhängigkeit von der zum Zeitpunkt der Blutentnahme vorliegenden Substanzmenge. Die Empfindlichkeit der verfügbaren Reagenzien ist hierbei sehr unterschiedlich. Ihre Empfindlichkeit der im jeweiligen Labor verwendeten Testmethoden muss deswegen lokal und ggf. mit dem Reagenzienhersteller geklärt werden. Gerinnungsglobaltests liefern meist nur im therapeutischen oder überdosierten Bereich semiquantitative Aussagen oder eine grobe Abschätzung, ob die Substanzen überhaupt im Plasma vorhanden sind.

Prinzipiell ist eine Messung der Medikamentenkonzentration bei DOAK keine Routine, zumal es bislang kaum Daten zu therapeutischen Bereichen oder zur klinischen Interpretation der gemessenen Plasmakonzentration gibt. Vor allem im Notfall bei einer akuten Blu- tung können aber quantitative Methoden zur Differenzialdiagnose beitragen:

- Zur Bestimmung der Dabigatran-Plasmakonzentration mit kalibrierter Thrombinzeit (z. B. HemoclotTest) wird die Messung der Thrombinzeit modifiziert, um das Messsignal auf die Einwirkung von Thrombininhibitoren zu konzentrieren. Durch die Verdünnung des Patientenplasmas werden Störeinflüsse vermindert und ein breiter Messbereich erreicht.

- Verfahren zur Bestimmung der Plasmakonzentration von Xa-Hemmern mit kalibrierter chromogener Anti-Faktor-Xa-Aktivität (Anti-Xa-Tests) wurden ursprünglich zur Überwachung der niedermolekularen Heparine entwickelt und sind für diese Indikation an vielen Stellen routinemäßig verfügbar. Mit spezifischen Kalibratoren sind diese auch für die direkten Anti-Xa-Hemmer geeignet und können quantitative Aussagen zur exakten Plasmakonzentration machen.

In jedem Fall muss der Einsatz hämostaseologischer Labormethoden zum Nachweis der DOAK den lokalen Gegebenheiten entsprechend geplant und zwischen Labor und Klinik abgestimmt werden. Diese Tests stehen nicht überall bzw. nicht rund um die Uhr zur Verfügung.

\section{Hämostaseologische bettseitige Messungen}

Eine weitere Ergänzung bieten bettseitig anwendbare Testverfahren, insbesondere die Thrombelastografie (TEG). Der Vorteil der modernen TEG ist neben der patientenseitigen Verfügbarkeit die Abbildung nahezu des gesamten Gerinnungsprozesses einschließlich der Quantifizierbarkeit mit Kenngrößen - entsprechend als Thrombelastometrie bezeichnet. Allerdings erfasst die TEG keine Störungen der Thrombozytenfunktion.

\section{Merke}

In-vitro-Testverfahren zur Messung der Thrombozytenfunktion liefern nur bei normaler Erythrozytenund Thrombozytenkonzentration gültige Ergebnisse, sodass sie bei vielen Intensivpatienten nicht eingesetzt werden können.

Die Domäne der TEG ist die Therapiesteuerung bei akuter Blutung, da die Interpretation der thrombelastografischen Messungen bei septischen Patienten bislang nicht ähnlich standardisiert möglich ist [13]. Aufgrund der hohen Kosten der Einzelmessung eignet sich die Thrombelastografie im Verlauf nur bedingt als Überwachungsinstrument.

Auch bei der TEG wird je nach Testansatz der extrinsische und intrinsische Weg getrennt ausgelöst (EXTEM und INTEM). Bei der Diagnostik bei akuter Blutung steht der EXTEM-Test im Vordergrund. Die Ergebnisse 
liefern unmittelbare Hinweise auf sinnvolle therapeutische Maßnahmen ( $\triangleright$ Abb. 3, $\triangleright$ Tab.6).

Modifikationen des Testansatzes ermöglichen eine weitere Unterscheidung zwischen spezifischen Ursachen einer Gerinnungsstörung, einer reduzierten MCF, einer Hyperfibrinolyse sowie der Heparinwirkung:

- Zur Differenzierung einer reduzierten MCF im EXTEM kann die Thrombozytenwirkung im Testansatz blockiert werden (FIBTEM). Nun bildet sich ein reiner Fibrinogen-Thrombus, der naturgemäß nur etwa ein Zehntel der Festigkeit eines Fibrinogen/Thrombozyten-Thrombus erreicht. Wird selbst dieser Wert unterschritten, liegt ein Fibrinogenmangel vor.

- Ob eine antifibrinolytische Therapie erfolgversprechend ist, kann mit dem APTEM beurteilt werden. Hierzu wird Aprotinin als Antifibrinolytikum zugesetzt. Fällt nun die Lyse im Vergleich zum EXTEM ganz oder geringer aus, erscheint die (ggf. wiederholte) Gabe eines Antifibrinolytikums indiziert.

- Zur Beurteilung einer Heparinwirkung dient das HEPTEM, bei dem der Zusatz einer Heparinase Heparin inaktiviert. Ist die CT im INTEM verlängert und im HEPTEM normal, so ist die verlängerte CT durch Heparin verursacht. Unter Protamin wird sich die CT im INTEM normalisieren.

\section{Hämostase bei akuter Blutung}

Viele Daten und Empfehlungen zur Gerinnungstherapie bei akuter Blutung stammen aus der Traumatologie. Tatsächlich ist das Polytrauma mit seiner massiven Gewebedestruktion und dem hämorrhagischen Schock ein hervorragendes Modell einer maximal aktivierten Gerinnungskaskade und der Entwicklung einer akuten Koagulopathie. Man geht davon aus, dass der systemische Schaden - charakterisiert vor allem durch Ischämie und Inflammation - wesentliche Voraussetzungen für eine akute Koagulopathie schafft ( $>$ Abb.4). In anderen Blutungssituationen, etwa bei Organperforation oder perioperativer Hämorrhagie mit Transfusionsbedarf sind häufig die Gewebszerstörung geringer und die Begleitumstände günstiger (z.B. besserer Erhalt der Normothermie), sodass hierbei eine größere Kompensationsbreite die Geschwindigkeit auf dem „Weg zur Koagulopathie“ verlangsamt [14].

\section{Thrombusbildung}

\section{Substrat für den Thrombus}

Der Effektor der Blutstillung ist die Bildung eines stabilen Thrombus am Ort der Blutung. Das oben skizzierte integrierte Modell der plasmatischen und zellulären Hämostase erklärt die lokale, hochkonzentrierte Thrombinfreisetzung als Grundlage hierfür. Gleichzeitig müssen die Bestandteile des Thrombus - namentlich Fibrinogen/Fibrin und Thrombozyten (sowie Ery-

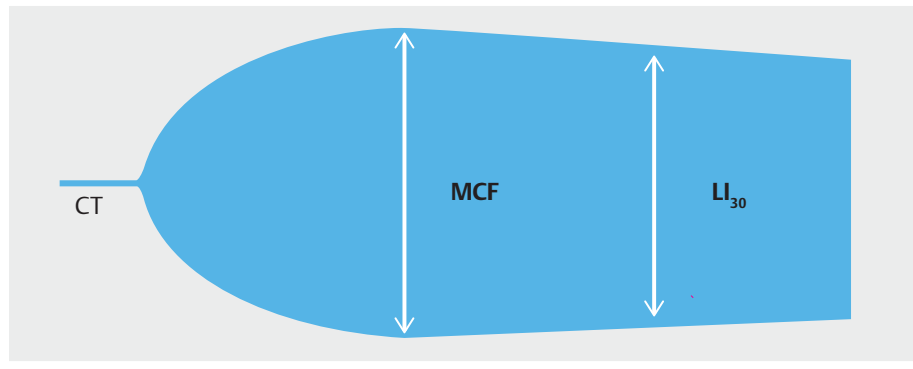

- Abb. 3 Kenngrößen der Thrombelastometrie im ROTEM-Verfahren.

- Tab. 6 Typische pathologische ROTEM-Befunde in akuten Blutungssituationen.

\begin{tabular}{|l|l|l|}
\hline Parameter & Wahrscheinliche Ursache & Therapie \\
\hline \multirow{2}{*}{$\mathrm{CT} \uparrow$} & $\begin{array}{l}\text { gestörte Initiierung der Gerinnung: } \\
\text { Faktoren des extrinsischen oder } \\
\text { intrinsischen Wegs (je nach gewähl- } \\
\text { tem Testverfahren) sowie X, V und II }\end{array}$ & PPSB, FFP \\
\hline INTEM-Test: Heparinwirkung & Protamin \\
\hline MCF $\downarrow$ & Fibrinogenmangel & $\begin{array}{l}\text { Fibrinogen- } \\
\text { konzentrat/FFP }\end{array}$ \\
\hline & Thrombopenie & $\begin{array}{l}\text { Thrombozyten- } \\
\text { Transfusion }\end{array}$ \\
\hline & & Tranexamsäure \\
\hline
\end{tabular}

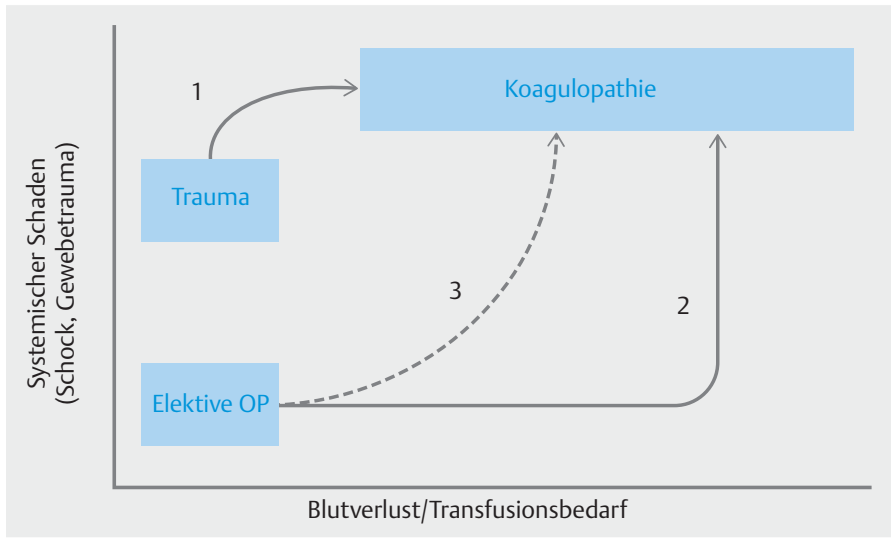

Abb. 4 Weg zur Koagulopathie (mod. nach [14]). Beim schweren Trauma besteht anfangs ein großer systemischer Schaden und die akute Koagulopathie entwickelt sich bereits früh, $d$. h. bereits nach vergleichsweise geringem Transfusionsbedarf (1). Umgekehrt kann etwa bei elektiver Operation trotz hohem Blutverlust oft die Homöostase gut aufrechterhalten werden - nicht zuletzt durch Transfusionen, sodass eine Dekompensation lange aufgehalten oder ganz vermieden werden kann. Die Koagulopathie entwickelt sich erst nach außergewöhnlich hohem Transfusionsbedarf (2). Diese Kompensationsbreite wird deutlich eingeschränkt, wenn die Operation selbst einen ausgeprägten proinflammatorischen Stimulus bietet, wie etwa bei herzchirurgischen Eingriffen mit langer extrakorporaler Zirkulation (3). 
throzyten) - in ausreichender Menge bereitstehen, um das häufig ja sogar makroskopisch erkennbare Gerinnsel zu bilden. Ausgerechnet die Fibrinogenreserve ist jedoch bei einer akuten Blutung am schnellsten verbraucht [15].

Eine frühzeitige Substitution von Fibrinogen und Thrombozyten bei akuter, massiver Blutung hat sich in vielen, vornehmlich allerdings retrospektiven Untersuchungen als effektiv erwiesen und daher Eingang in die Leitlinien gefunden $[16,17]$. Da die Gabe von Vollblut in Mitteleuropa obsolet ist, verabreicht man Blutkomponenten oder Faktorenkonzentrate. Im militärischen Bereich dagegen erlebt die Transfusion frischen Vollbluts vorwiegend aus logistischen Gründen eine Renaissance mit exzellenter hämostaseologischer Wirksamkeit [1]. Bei der Transfusion von Komponenten führt ein Verhältnis zwischen Erythrozytenkonzentrat und Frischplasma von 1:1 zu einer effektiveren Blutstillung und zu einer tendenziell, aber nicht signifikant verbesserten Mortalität im Vergleich zu einer 2:1Transfusion [18].

Merke

Bei Massivtransfusionen sollte man ein Verhältnis von 1:1:1 zwischen Erythrozyten-, Frischplasma- und Thrombozytenkonserven anstreben.

Die vorgeschalteten Faktoren der Gerinnungskaskade stehen meist in ausreichender Menge und Aktivität zur Verfügung-zumindest bei nicht medikamentös antikoagulierten Patienten (Ausnahmen s.u., Abschnitt „Immunkoagulopathien und andere erworbene hämostaseologische Störungen“). Daher ist auch die Substitution von PPSB in akuten Blutungssituationen bei nicht antikoagulierten Patienten keine erstrangige Maßnahme.

\section{Fibrinogen}

Fibrinogen kann entweder als natürlicher Bestandteil von Plasmakonserven (FFP, lyophilisiertes Plasma) oder als Konzentrat verabreicht werden. Angestrebt wird zumindest ein Erhalt der unteren physiologischen Konzentration um $150-200 \mathrm{mg} / \mathrm{dl}$ [17]. Ob ein höherer Zielwert oder gar superphysiologische Konzentrationen tatsächlich zu einer verbesserten Hämostase führen - oder andererseits zu vermehrten thromboembolischen Komplikationen - ist noch umstritten. Zumindest bei geburtshilflichen Blutungskomplikationen scheinen Fibrinogenkonzentrationen von über 200 $\mathrm{mg} / \mathrm{dl}$ von Vorteil zu sein $[19,20]$.

Es hängt stark von der klinischen Situation ab, ob durch die Transfusion von FFP alleine ein ausreichender Anstieg der Fibrinogenkonzentration erreicht werden kann.

\section{Merke}

Um bei einem $70 \mathrm{~kg}$ schweren Patienten einen Anstieg der Fibrinogenkonzentration um $100 \mathrm{mg} / \mathrm{dl}$ zu erreichen, ist die Transfusion von etwa 1000 ml FFP (entsprechend 4 Transfusionseinheiten) oder die Substitution von 3-4g Fibrinogen in konzentrierter Form erforderlich.

Häufig wird daher die Gabe von Fibrinogenkonzentrat schneller zur Wiederherstellung des erforderlichen Fibrinogenspiegels führen und damit zur Durchbrechung der Koagulopathie beitragen. Zudem kann damit eine möglicherweise drohende Volumenüberladung vermieden werden. Leider fehlen hierzu aber hochwertige kontrollierte Studien [21]. Im weiteren Verlauf der intensivmedizinischen Therapie kann man fast immer mit einem spontanen Anstieg der Fibrinogenkonzentration innerhalb von Stunden bis Tagen rechnen.

\section{Thrombozyten}

Thrombozyten stehen hierzulande überwiegend als Apherese-Konzentrate von Einzelspendern zur Verfügung, aber auch als gepoolte Konzentrate aus mehreren Spenden. Angestrebt wird bei einer Blutung eine Thrombozytenkonzentration von mindestens 50G/I bzw. von mindestens $100 \mathrm{G} / \mathrm{l}$ bei ZNS-Blutungen oder bei zunächst therapierefraktären Blutungen. Diese Empfehlungen basieren auf Erfahrungen aus der Traumatologie, lassen sich aber auch auf andere Blutungsauslöser übertragen.

Dennoch sollte vor allem wegen der hohen Immunisierungsrate die Transfusion von Thrombozyten streng indiziert werden, da sonst rasch eine Transfusionsrefrakterität droht. Weitere Probleme sind die begrenzte Verfügbarkeit sowie drohende thromboembolische Komplikationen. Daher ist eine prophylaktische Transfusion bei Thrombopenie ohne akute Blutung in der Regel erst beim Unterschreiten einer Thrombozytenkonzentration von 10-15G/l sinnvoll, da erst darunter eine Zunahme der Rate spontaner Blutungen droht [22].

\section{latrogene Koagulopathie}

Eine massive kristalloide und/oder kolloidale Volumensubstitution kann die traumatische Koagulopathie noch verstärken, da sie ihrerseits zu einer Verdünnung oder partiellen Inaktivierung der plasmatischen und zellulären Gerinnungsfaktoren führt. Zudem kann sie zu einer Verschlechterung der Hypothermie und der Azidose beitragen, Letzteres zumal vor der Ära der balancierten Infusionslösungen.

Daher wird häufig die „lethal triade“ (Koagulopathie, Azidose, Hypothermie) konzeptionell um die Volumentherapie zu einem „tödlichen Quartett“ ergänzt ( Abb. 1) [23]. Allerdings fällt es schwer, die Volumentherapie unabhängig von anderen, nicht direkt modifi- 
zierbaren Einflüssen (Verletzungsschwere, Schock, prähospitale Versorgungs- und Transportzeiten) als Faktor zu identifizieren, der das Outcome verschlechtert. Umgekehrt unterstreichen die Daten-insbesondere aus dem Traumaregister der Deutschen Gesellschaft für Unfallchirurgie-die große Bedeutung einer frühzeitigen und gezielten Transfusions- und Substitutionstherapie unmittelbar nach der Krankenhausaufnahme.

Merke

Schwere Azidose $(\mathrm{pH}<7,2)$ und Hypothermie sind Co-Faktoren jeder Koagulopathie.

Ein weiterer, iatrogen bedingter Komplikationsfaktor ist die massive Transfusion von Erythrozytenkonzentraten. Als Massivtransfusion wird meist eine Transfusion von mehr als 10 Konzentraten in 24 Stunden bezeichnet, aber die Definition ist nicht einheitlich. Eine hohe Menge rasch transfundierter Erythrozytenkonzentrate kann einerseits zu Gerinnungsstörungen aufgrund einer transfusionsbedingten Hypokalzämie führen, andererseits wirkt der hohe Eintrag von Zellzerfallsprodukten aus länger gelagerten Konzentraten proinflammatorisch und verstärkt die Koagulopathie zusätzlich.

Merke

Die Serumkonzentration von $\mathrm{Ca}^{2+}$ sollte um oder über $0,9 \mathrm{mmol} / \mathrm{l}$ gehalten werden. Die Forderung nach bzw. der gezielte Einsatz durchwegs kürzer gelagerten Erythrozytenkonzentrate ist angesichts der Knappheit der Ressource Blut unrealistisch.

\section{Thrombozytäre Funktion bei akuter Blutung}

\section{Pathophysiologie}

Das oben skizzierte Hämostasemodell integriert nicht nur den ex- und intrinsischen Weg der plasmatischen Gerinnung, sondern auch die zelluläre, d. h. thrombozytäre Gerinnung [24]. Tatsächlich spielt die anfangs sehr rasch erfolgende Thrombozytenaktivierung (,schnelle Gerinnung“) nicht nur eine Rolle hinsichtlich des eigentlichen Verschlusses der Blutungsquelle, sondern vor allem auch hinsichtlich der Lokalisierung der Gerinnungsvorgänge: Thrombozyten schwimmen durch den Fåhræus-Lindqvist-Effekt im langsameren Randstrom (kleinerer) Gefäße (Margination), um am Ort einer potenziellen Läsion zu adhärieren, gleichzeitig aktiviert zu werden und sich zu konzentrieren ( $\mathbf{A} \mathbf{b} \mathbf{b} \mathbf{b} \mathbf{5})$. Da vor allem die Bildung des Prothrombinase-Komplexes aus den Faktoren X und V durch die Thrombozytenoberfläche katalysiert wird, ist unter idealen Bedingungen eine hohe Ortsselektivität der plasmatischen Gerinnungsvorgänge gewährleistet.

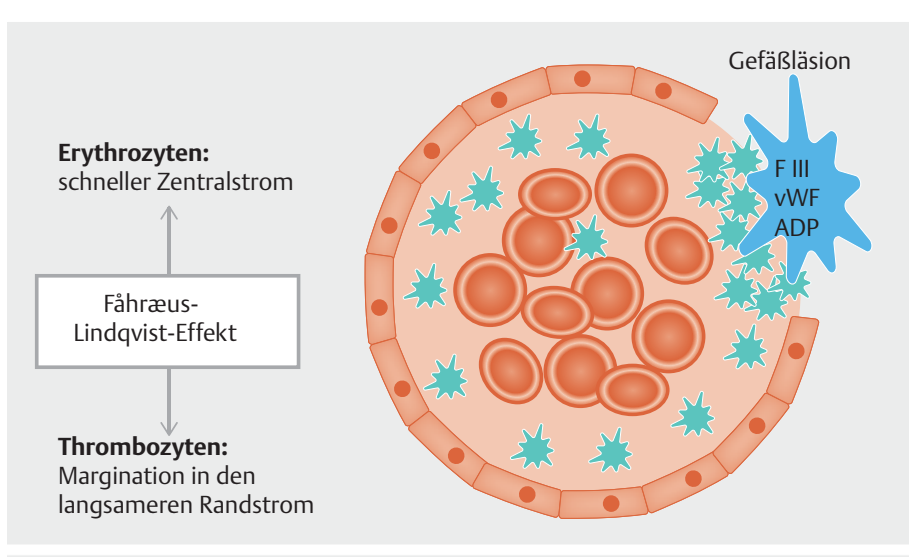

- Abb. 5 Mechanismen der Thrombozytenmargination und -aktivierung bei endothelialen Schäden.

\section{Therapiemöglichkeiten}

Die Standardtherapie der quantitativen Thrombopenie und der funktionellen Thrombopathie einschließlich der Thrombozytenaggregationshemmung ist die Transfusion von Thrombozytenkonzentraten.

Desmopressin ist ein Vasopressin-Analogon mit reduzierter vasokonstriktorischer Aktivität. Seine erwünschte prokoagulatorische Wirkung besteht in der Freisetzung von von-Willebrand-Faktor (vWF) und von Faktor VIII aus Weibel-Palade-Körperchen.

\section{Merke \\ Die Wirkung von Desmopressin ist erschöpflich und eine Wiederholung frühestens nach 24 Stunden sinn- voll. Die Einzeldosis beträgt $0,3-0,4 \mu \mathrm{g} / \mathrm{kgKG}$.}

Allerdings hat Desmopressin auch antikoagulatorische Eigenschaften durch Freisetzung von Prostazyklin und „tissue plasminogen activator“. Entsprechend scheint seine hämostaseologische Wirkung durch den gleichzeitigen Einsatz von Antifibrinolytika optimiert zu werden. Als ADH-Analogon kann es eine Flüssigkeitsretention verstärken. Andererseits kann es bei rascher Injektion zu einer Hypotonie führen.

Die gesicherten Indikationen sind in erster Linie die Behandlung des von-Willebrand-Syndroms und die partielle Antagonisierung einer ASS-Wirkung. Diese Antagonisierung funktioniert jedoch nach Anwendung anderer Thrombozytenaggregationshemmer wie Clopidogrel nicht effektiv. Nach herzchirurgischen Operationen kann Desmopressin den Transfusionsbedarf senken, vor allem bei Patienten mit reduziertem vWF-Spiegel [25]. 


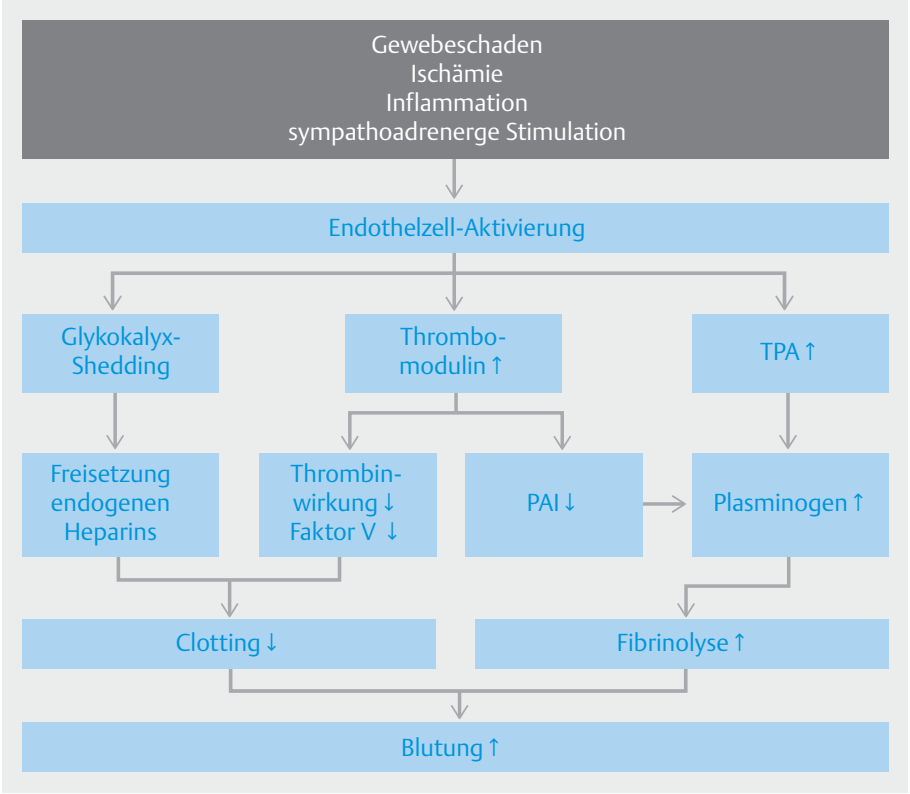

- Abb. 6 Folgende Komponenten tragen zur Entwicklung einer Hyperfibrinolyse bei: Im Schock kommt es zu einer verstärkten Expression von endothelständigem Thrombomodulin, das Thrombin bindet und damit hinsichtlich seiner prokoagulatorischen Aktivität inaktiviert. Gleichzeitig wird der thrombinaktivierte Fibrinolyseinhibitor (TAFI) gehemmt. Zudem aktiviert der Thrombin/Thrombomodulin-Komplex die physiologischen Antikoagulanzien Protein C und Protein S, die ihrerseits sowohl prokoagulatorische (Faktor V und VIII) als auch antifibrinolytische Faktoren (PAI 1) hemmen.

\section{Fibrinolytische Aktivität}

\section{Physiologische Fibrinolyse}

Dem physiologischen Gerinnungssystem steht das System der physiologischen Fibrinolyse gegenüber. Seine zentralen Funktionen sind die Begrenzung der Thrombusbildung auf den Ort und die Dauer der Blutung sowie wichtige immunologische, dabei vor allem antiinflammatorische Effekte. Prinzipiell wird das fibrinolytische System gleichzeitig mit der Hämostase aktiviert, da der freie Plasminogen-Aktivator bei Anwesenheit von Fibrin-also am Thrombus - Plasminogen zu Plasmin als wesentlichem fibrinolytischem Enzym aktiviert. Dieser profibrinolytische Weg wird durch den thrombinaktivierten Fibrinolyseinhibitor (TAFI) gehemmt, sodass die Thrombusbildung bei hoher Thrombinaktivität - also in der Phase der akuten Blutstillung-möglichst ungestört ablaufen kann. Ein weiterer potenter antifibrinolytischer Faktor ist Faktor XIII.

\section{Hyperfibrinolyse}

Dennoch kann eine überschießende Aktivierung der physiologischen Fibrinolyse auftreten (Hyperfibrinolyse), die zu einer wesentlichen und bereits früh einsetzenden Verstärkung der traumatischen Koagulopathie führt. Eine wesentliche Ursache ist, dass der endothelial präformierte „tissue plasminogen activator“ (tPA) unmittelbar beim Eintritt des Traumas freigesetzt wird, während der "plasminogen activator inhibitor“ (PAI 1) erst noch gebildet werden muss. Dies alleine erklärt aber eine schwere systemische Fibrinolyse nur unzureichend.

Weitere Komponenten der akuten Hyperfibrinolyse sind in $>$ Abb. $\mathbf{6}$ dargestellt. Durch die massive Fibrinolyse steht nun nicht mehr ausreichend Fibrinogen zur Verfügung, das bei diesem hohen Umsatz als Substrat für den „erneuten“ Thrombusaufbau dringend erforderlich ist. Dieser Mangel führt zum Vollbild der Verbrauchskoagulopathie.

\section{Diagnostik und Therapie}

Hinsichtlich der Diagnostik und Therapie der traumatisch bedingten Hyperfibrinolyse stehen mit der Thrombelastografie und der Gabe von Antifibrinolytika, namentlich Tranexamsäure [27], etablierte Methoden zur Verfügung. $\varepsilon$-Aminokapronsäure kann alternativ verwendet werden. Das Risikoprofil von Aprotinin, einem weiteren Antifibrinolytikum, dagegen scheint deutlich ungünstiger.

\section{Merke}

Bei Traumapatienten wird empfohlen, so früh wie möglich, also noch prähospital, eine Loading-Dose von $1 \mathrm{~g}$ Tranexamsäure zu verabreichen, gefolgt von einer Infusion von $1 \mathrm{~g}$ über die 8 Stunden nach Krankenhausaufnahme [28]. Diese und ggf. weitere Gaben können mit der Thrombelastografie gesteuert werden. In Analogie erscheint der Einsatz von Tranexamsäure auch beim Verdacht auf bzw. nachgewiesener Hyperfibrinolyse aus anderen Ursachen sinnvoll.

Neueste Daten weisen aber darauf hin, dass die Inzidenz der traumatisch bedingten Hyperfibrinolyse möglicherweise überschätzt wird [29]: Bei über 2500 schwer verletzten Patienten zeigten $18 \%$ in einer binnen einer Stunde nach Krankenhausaufnahme angefertigten Thrombelastografie eine Hyperfibrinolyse (Mortalität in dieser Gruppe: 34\%) und 36\% eine physiologische Fibrinolyse (Mortalität: 14\%). Bei der mit $46 \%$ größten Gruppe aber war ein komplettes Erliegen der fibrinolytischen Aktivität nachweisbar (Mortalität: $23 \%)$. Während sich die Gruppen hinsichtlich der Verletzungsschwere und -lokalisation, initialer Hb-Konzentration und INR nicht unterschieden, hatten die Patienten mit Hyperfibrinolyse häufiger penetrierende Verletzungen und bei Aufnahme einen niedrigeren Blutdruck. Aufgrund des hohen mit der Hyperfibrinolyse assoziierten Mortalitätsrisikos, des günstigen Sicherheitsprofils von Tranexamsäure und der eindeutigen Studienlage sollte aber bis auf Weiteres nicht von der oben genannten Empfehlung zur antifibrinolytischen Therapie abgewichen werden. 


\section{Hämostase bei Sepsis und septischem Schock}

\section{Pathophysiologie}

In der Sepsis wird die Gerinnungskaskade zunächst über ganz ähnliche Mechanismen angestoßen wie bei der akuten Blutung: Die durch die Infektion aktivierten Makrophagen und Monozyten setzten - analog zu den Endothelzellen nach einem Gefäßtrauma - Gewebethromboplastin frei (syn: „tissue factor“, Faktor III). Dies löst im Wesentlichen 3 Mechanismen aus ( Abb. 7):

- Durch die thrombozytäre Aktivierung werden Adhäsionsmoleküle - vor allem P-Selektin (P steht für „platelet“) - exprimiert, die ihrerseits die leukozytäre Aktivierung verstärken und damit unter anderem als positive Rückkoppelung wiederum die Gewebethromboplastin-Freisetzung steigern.

- Durch die Aktivierung der Gerinnungskaskade wird Thrombin freigesetzt, das unmittelbar zur Thrombusbildung führt. Da inflammationsbedingt das Endothel buchstäblich ubiquitär aktiviert ist (und nicht nur am Ort einer abgegrenzten Läsion), kommt es zur disseminierten intravasalen Gerinnung (DIC).

- Die DIC läuft umso stärker ab, als wesentliche antithrombotische Mechanismen in der Sepsis - anders als bei der akuten Blutung - nicht ebenso stark wie die proinflammatorische Kaskade aktiviert werden: Durch den septischen Endothelzellschaden einschließlich der früh einsetzenden Apoptose wird als ein wesentlicher Faktor aus dem Endothel weniger Thrombomodulin freigesetzt, das eigentlich die thrombolytische Gegenreaktion anstößt. Dadurch kommt es zu einem Versagen des Protein-C-Systems.

\section{Symptomatik}

Klinisch eindeutige Manifestationen der DIC in der Sepsis sind Hautnekrosen und digitale Nekrosen an Händen und Füßen, seltener auch von Ohren, Nase und Mamillen. Als einzelne Krankheitsbilder sind das Waterhouse-Friderichsen-Syndrom und das Toxic-Shock-Syndrom zu nennen.

Andere Manifestationen treten schwerer identifizierbar als Komponenten des Multiorganversagens auf, etwa im Rahmen eines ARDS, eines akuten Nierenversagens oder einer septischer Enzephalopathie. Daher wird auch zwischen verdeckter und offensichtlicher DIC unterschieden, wobei ein Übergang hin zur offensichtlichen DIC möglich ist. Um vor allem wegen dieser Gefahr auch eine verdeckte DIC mit ausreichender Sensitivität identifizieren zu können, wurden mehrere Scoring-Systeme entwickelt (Übersicht bei [26]).

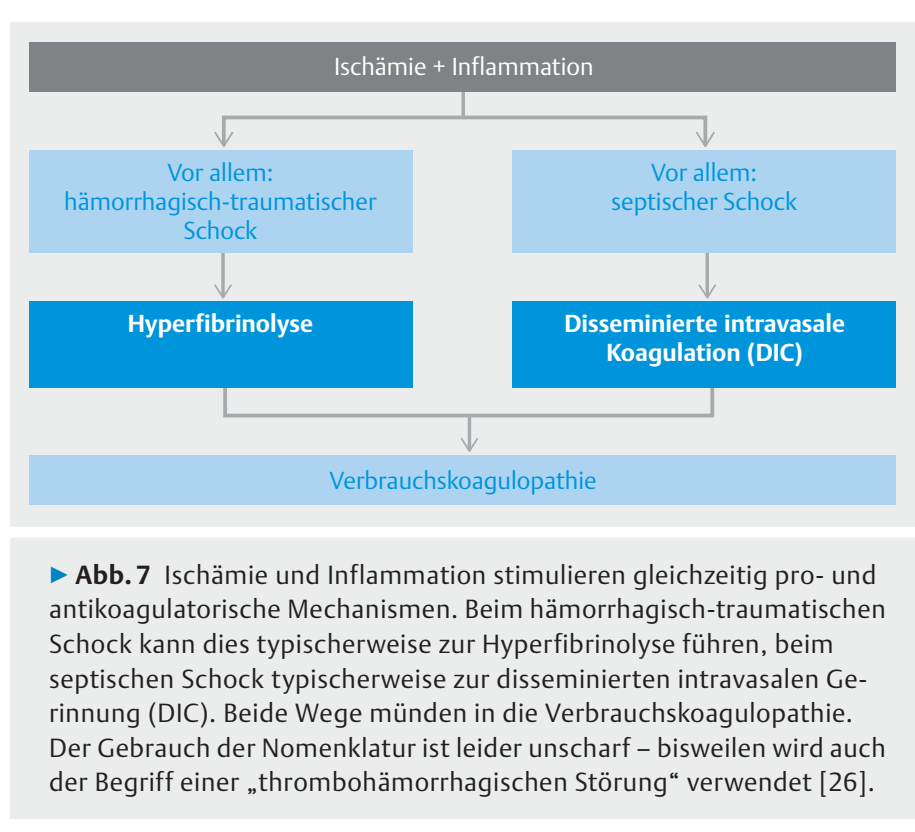

\section{Thrombopenie in der Sepsis}

Häufig besteht bei akut und vor allem kritisch kranken Patienten eine Thrombopenie, die ätiologisch nicht immer auf den ersten Blick einzuordnen ist und die vor allem bei Thrombozytenzahlen unter 50-20.000 G/I eine Störung der primären Hämostase verursachen.

Die Differenzialdiagnose der Thrombozytopenie ist oft ein Thema in der Intensivmedizin. Aufwendige serologische Untersuchungen im immunhämatologischen Labor ermöglichen es, Autoantikörper gegen Thrombozyten zu spezifizieren. Meist besteht aber „nur“ eine Thrombopenie, sehr selten sind (abgesehen von der heparininduzierten Thrombopenie, auf die hier nicht weiter eingegangen wird) thrombozytäre Autoantikörper auch mit einer Funktionseinschränkung verbunden. - Abb. 8 zeigt ein pragmatisches Vorgehen bei Thrombopenie in Akutsituationen.

\section{Therapie der Gerinnungsstörung in der Sepsis}

\section{Basistherapie: konventionelle Antikoagulation}

Der Kern der Therapie einer septischen DIC ist die Antikoagulation, da es keine etablierten Verfahren gibt, die den zugrunde liegenden Endothelschaden abschwächen könnten. Wenn nicht durch eine Blutung kontraindiziert, sollte man bei septischen Patienten frühzeitig mit einer Antikoagulation beginnen [26]. Theoretisch wäre die Verwendung eines Thrombinantagonisten zur Antikoagulation ideal, letztlich hat sich aber die Nutzung von unfraktioniertem oder niedermolekularem Heparin bewährt. Unser Vorgehen ist in $>$ Abb. 9 dargestellt. 


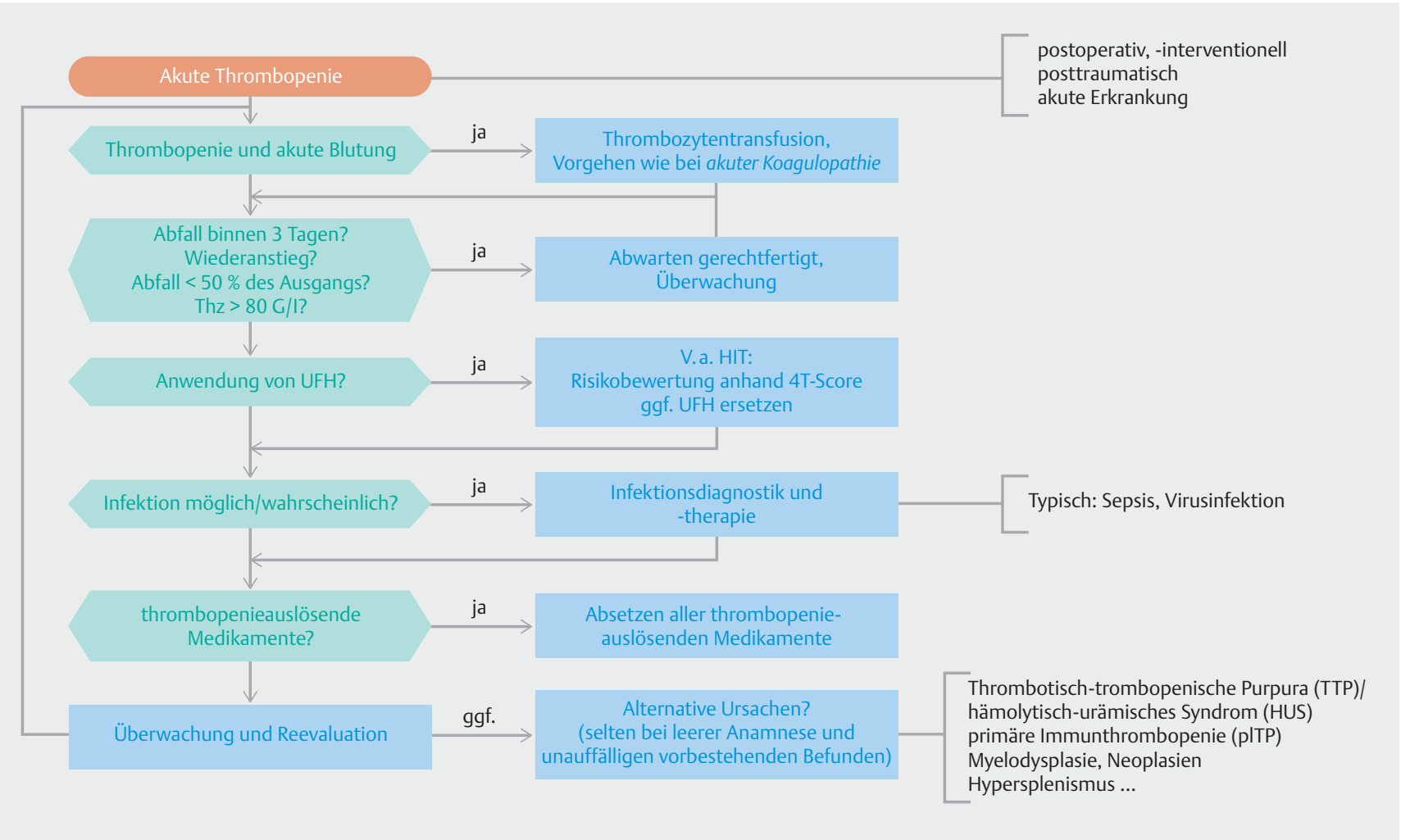

- Abb. 8 Pragmatisches Vorgehen bei akuter Thrombopenie. (UFH: unfraktioniertes Heparin; HIT: heparininduzierte Thrombopenie Typ II). Typische medikamentöse Auslöser einer Thrombopenie sind neben verschiedenen Antibiotika und Virostatika unter vielen anderen Antiepileptika und verschiedene Nicht-Opiat-Analgetika.

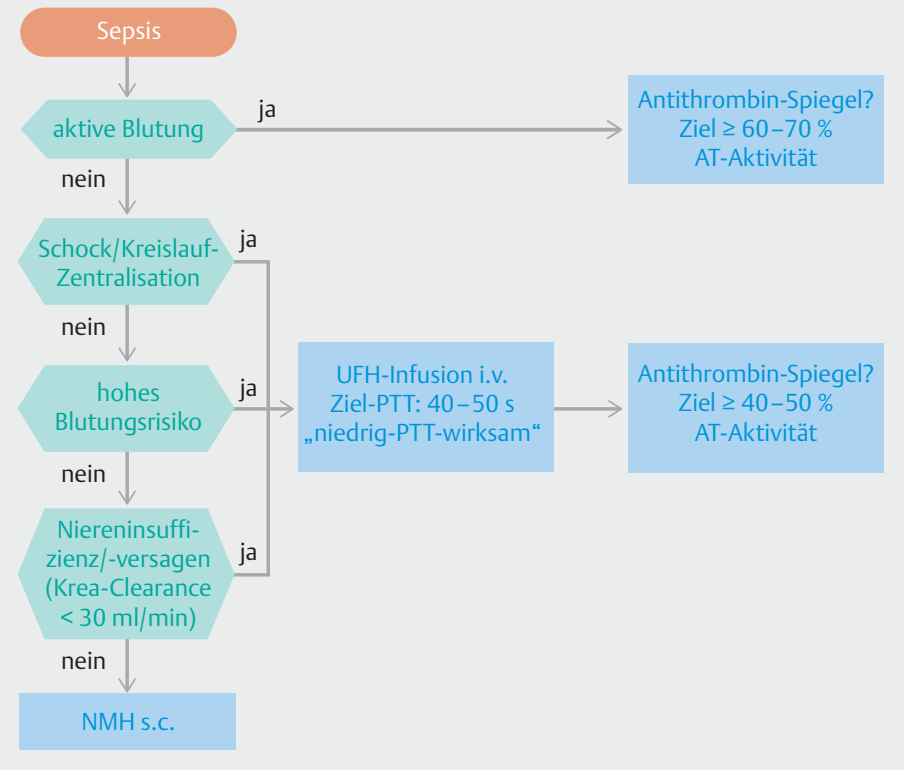

- Abb.9 Pragmatisches Vorgehen zur Antikoagulation septischer Patienten.
Eine Reihe weiterer Therapieansätze wurde wissenschaftlich untersucht, konnte sich aber aufgrund der Studienlage nicht durchsetzen:

\section{Antithrombin}

Die hochdosierte Antithrombingabe (erreichte Aktivität im Mittel 180\%) wurde in den späten 1990er Jahren in der als klassisch geltenden KyberSept-Studie an über 2300 Patienten mit schwerer Sepsis oder im septischen Schock randomisiert gegenüber Placebo untersucht [30]. Die Kombination aus hochdosiertem Antithrombin und Heparin bot keinen Überlebensvorteil gegenüber Placebo mit oder ohne Heparin, zeigte aber eine erhöhte Blutungsinzidenz. Bei Patienten, die kein Heparin erhielten, zeigte die Antithrombingabe einen Überlebensvorteil gegenüber Placebo. Die Heparingabe (UFH oder NMH s. c. zur Thromboseprophylaxe) war im Studiendesign aber weder kontrolliert noch standardisiert.

Niedriger dosiertes Antithrombin (erreichte Aktivität um $110 \%$ ) in Kombination mit Heparin führte in kleinen Studien zu einer Besserung einer DIC anhand von Surrogat-Parametern, schlug sich aber nicht auf das Outcome der Patienten nieder [31]. 
Die Antithrombinspiegel, die wir bei unseren vorwiegend operativen Patienten anstreben ( $\downarrow$ Abb.9), liegen aus Gründen des Risiko/Nutzen-Profils und der Pharmaökonomie niedriger.

\section{Merke}

Eine effektive Antikoagulation mit Heparin (UFH oder $\mathrm{NMH}$ ) ist die Antikoagulation der Wahl bei septischen Patienten, niedrige Antithrombinspiegel sollte man aber substituieren.

\section{Aktiviertes Protein C}

Einen wesentlichen Beitrag zur septischen Gerinnungsstörung im Sinne einer Hyperkoagulopathie liefert das Versagen des Protein-C-Systems. Ursache hierfür ist vor allem der Mangel an Thrombomodulin. Daraus ergeben sich 2 Therapieansätze, nämlich die Substitution von Protein C oder von Thrombomodulin.

Rekombinantes aktiviertes Protein C war bis 2012 knapp 10 Jahre lang unter dem Handelsnamen Xigris erhältlich. Um dieses Präparat entwickelte sich nach einem anfänglichen Hype ein wahrer Glaubenskrieg, teilweise fernab eines wissenschaftlichen Fundaments [32]. Letztlich zeigte das Präparat bei zunehmender Indikationsausweitung hin zu septischen Patienten mit geringeren Organkomplikationen eine geringe oder nicht nachweisbare Effektivität, während es - wenig überraschend - Blutungskomplikationen verursachen konnte [33]. Auf eine Empfehlung der FDA hin zog der Hersteller das Präparat schließlich vom Markt zurück.

Wir bedauern diesen kompletten Rückzug, den wir für ebenso wenig sinnvoll halten wie die zuvor verfolgte aggressive Marketing- und Hochpreisstrategie des Herstellers. Nach den wissenschaftlichen Ergebnissen und eigenen Erfahrungen hat durchaus ein Teil der Patienten von dem Präparat profitiert, insbesondere schwer kranke erwachsene Patienten in der Frühphase eines septischen Schocks, die rasch ein Mehrorganversagen mit DIC entwickelt hatten und während der Therapie eine ausreichende Thrombozytenzahl aufwiesen - zugegebenermaßen eine kleine Subgruppe.

\section{Thrombomodulin}

Weiter am Beginn der Kaskade reduziert Thrombomodulin die prothrombotische Wirkung von Thrombin zugunsten der Aktivierung des Protein-C-Systems. Tatsächlich ist rekombinantes humanes Thrombomodulin in Japan pharmakologisch verfügbar und seine Anwendung zeigt vielversprechende, aber noch nicht abschließend beurteilbare Resultate [34].

\section{Immunkoagulopathien und andere erworbene hämostaseologische Störungen}

Wir konzentrieren uns hier auf akute Erstmanifestationen erworbener Hämostasestörungen und verzichten auf die Darstellung der Therapie chronischer, fast immer anamnestisch bekannter und häufig hereditärer Gerinnungsstörungen (z. B. klassische Formen der Hämophilie, von-Willebrand-Syndrom).

\section{Faktor-XIII-Mangel}

Faktor XIII ist der letzte in der klassischen Gerinnungskaskade wirkende prokoagulatorische Faktor: Entsprechend seines Namens (fibrinstabilisierender Faktor) stabilisiert er das Fibrinnetzwerk vor allem gegen die endogene Plasminwirkung. Damit und darüber hinaus richtet sich seine Wirkung bereits auf die folgende Wundheilung und Infektabwehr [35].

\section{Klinik und Diagnose}

Die Aktivität von Faktor XIII wird durch die hämostaseologischen Globaltests nicht erfasst und auch die Thrombelastografie ist hinsichtlich eines Faktor-XIIIMangels nicht ausreichend sensitiv [36]. Daher wird oft ein klinischer Verdacht einer gezielten Diagnostik mit der Messung der Faktor-XIII-Aktivität vorangehen.

\footnotetext{
Merke

Die typische Symptomatik des Faktor-XIII-Mangels sind unerklärte Blutungen, insbesondere in Form von Nachblutungen einige Stunden nach OP-Ende. Bei vorbestehender Aktivierung des Gerinnungssystems wie etwa bei Tumorpatienten kann sich die Blutungsneigung bereits früher, z. B. schon intraoperativ manifestieren [37].
}

\section{Substitution}

Klare Grenzwerte für die Indikation zur Faktor-XIII-Substitution in der Akutsituation sind nicht etabliert. Kontrollierte Studien zur Wirksamkeit fehlen weitgehend. In 2 kleineren, kontrollierten und randomisierten Studien an herzchirurgischen Patienten erwies sich eine prophylaktische Substitution von Faktor XIII als ineffektiv hinsichtlich des postoperativen Transfusionsbedarfs $[38,39]$. In der Post-hoc-Analyse der Studie von Godje et al. zeigten allerdings die Patienten mit einer erniedrigten Faktor-XIII-Aktivität (studiendesignbedingt unabhängig von einer möglichen Substitution) einen erhöhten Transfusionsbedarf.

Namentlich bei einer klinisch persistierenden diffusen Blutungsneigung zusammen mit einer erniedrigten Faktor-XIII-Aktivität kann eine Substitution mit FaktorXIII-Konzentraten (human oder rekombinant) erwogen werden. 
Hinsichtlich der therapeutischen Wirkung bei der Wundheilung sind dringend kontrollierte Studien erforderlich.

\section{Erworbene Hemmkörper-Hämophilie}

Das spontane oder durch Systemerkrankungen induzierte Auftreten von Autoantikörpern gegen körpereigene oder transfundierte Antigene spielt in der klinischen Hämostaseologie eine wichtige Rolle. Vor allem Antikörper gegen die Faktoren des intrinsischen Systems - am häufigsten gegen Faktor VIII (HemmkörperHämophilie), seltener auch gegen Faktor IX und XIkönnen zu schweren Blutungskomplikationen vor allem im fortgeschrittenen Alter oder auch bei Frauen (z. B. postpartal) führen. Diagnostik und Therapie dieser erworbenen und oft schwer bis lebensbedrohlich verlaufenden Blutungsneigung sind außerordentlich aufwendig.

\section{Diagnose}

Der Verdacht sollte sich auf eine Hemmkörper-Hämophilie richten, wenn trotz optimaler Gerinnungsbehandlung eine diffuse, multilokale Blutungsneigung als Ausdruck einer Koagulopathie sowie die Verlängerung der aPTT persistieren. Etwa ein Drittel der Patienten hat Risikofaktoren wie Rheuma, Malignome, eine systemischen Lupuserkrankung oder eine vorangegangene Entbindung. Bemerkenswert ist, dass die postpartale Latenz im Median bei 77 Tagen liegt (IQR: 15 - 117) [40]. Bei zwei Dritteln der Patienten tritt die Hemmkörper-Hämophilie aber idiopathisch auf.

Diagnostisch stehen 3 Ansätze im Vordergrund:

- Die Akutdiagnostik gelingt mit dem Plasmaaustauschtest: Die aPTT ist bei Hemmkörper-Hämophilie stark verlängert. Zusätzlich benötigt man eine Probe eines Probanden mit normaler aPTT. Nun wird Plasma des Verdachtspatienten mit Probandenplasma gemischt und erneut die aPTT bestimmt, die mit hoher Wahrscheinlichkeit normal sein wird. Das gemischte Plasma wird für eine Stunde bei $37^{\circ} \mathrm{C}$ inkubiert. Eine nun aufgetretene Verlängerung der aPTT in einer erneuten Messung gilt als starker Hinweis auf das Vorhandensein von Antikörpern, da diese nach der Inkubation auch die Gerinnungsfähigkeit des Probandenbluts inhibieren.

Zwar ist der negative Vorhersagewert dieses Tests akzeptabel, aber er liefert potenziell auch eine hohe Zahl falsch positiver Ergebnisse. Daher sind weitere Untersuchungen zur Bestätigung der Diagnose unumgänglich:

- Der Antikörper kann im Bethesda-Test direkt nachgewiesen und mit Titer-Bestimmung quantifiziert werden.

- Die Aktivitätsmessung des inhibierten Faktors steht relativ unkompliziert vor allem als Verlaufsüberwachung zur Verfügung. Sie ist in der foudroyanten
Phase der Hemmkörper-Hämophilie meist auf unter $10 \%$, oft auf nur $1-2 \%$ reduziert.

\section{Therapie}

Durch jede Hämophilie fällt in der akuten Hämostase die positive Rückkopplung in der Aktivierung der Gerinnungskaskade weg.

\section{Merke}

Bei der Hemmkörper-Hämophilie ist eine Substitution des betroffenen Faktors - sei es als Faktorenkonzentrat, sei es als FFP - streng kontraindiziert, da hierdurch die Expression des Hemmkörpers zusätzlich angeregt würde.

Durch eine superphysiologische Anregung des klassischen extrinsischen Wegs wird die Rückkopplung über den intrinsischen Weg verzichtbar. Dazu muss aktivierter Faktor VII in hoher Konzentration verfügbar sein. Hierzu stehen 2 Präparate klinisch zur Verfügung: rekombinanter aktivierter Faktor VII (NovoSeven) und ein PPSB-Präparat mit Faktor VII in aktivierter Form (FEIBA). Beide Präparate gehen mit einem hohen Thrombembolierisiko einher und sind teuer. Eine Überlegenheit eines dieser Präparate über das andere hinsichtlich Effektivität und Sicherheit konnte nicht nachgewiesen werden. Als ultima ratio können die beiden Präparate ggf. auch kombiniert angewendet werden.

Zur raschen Entfernung der pathologischen Autoantikörper ist auch der erfolgreiche Einsatz der therapeutischen Plasmapherese oder von Immunadsorptionsverfahren beschrieben. Sofern in der klinischen Routine verfügbar, ist damit eine rasche und kosteneffektive Reduktion der pathologischen Antikörper möglich. Damit lässt sich die trotzdem konsequent erforderliche gerinnungsaktivierende Medikation deutlich reduzieren.

Ein alternativer neuer Therapieansatz ist die Gabe von rekombinantem porcinem Faktor VIII (Obisur), der annähernd die gleiche gerinnungsphysiologische Wirkung wie der humane Faktor hat, aufgrund seiner anderen Struktur aber nicht vom humanen Autoantikörper angegriffen wird [41].

Neben der Gerinnungstherapie ist eine mehrwöchige Immunsuppression bis zur Remission das zweite Standbein der Therapie. Hierfür wird ein Schema der Gesellschaft für Thrombose- und Hämostaseforschung verwendet, dass auf dem sequenziellen Einsatz von Steroiden, Cyclophosphamid und Rituximab basiert. Die erfolgreiche Remission ist prognostisch bestimmend. 


\section{Erworbenes von-Willebrand-Syndrom}

Die Diagnosestellung eines erworbenen von-Willebrand-jürgens-Syndroms (avWD) ist komplizierter als die einer erworbenen Hämophilie. Die Pathogenese der Erkrankung und die Diversität der beteiligten Mechanismen wurden in den vergangenen Jahren deutlich. Bei vielen Patienten gibt es keinen immungenetischen Auslöser - im Gegensatz zur erworbenen Hämophilie, bei der dieser eine zentrale Rolle spielt. Es besteht ein klarer Bedarf für verbesserte diagnostische und differenzialtherapeutische Möglichkeiten für das avWD.

Aufgrund der eingeschränkten diagnostischen Möglichkeiten ist das avWD insgesamt unterdiagnostiziert. Routinelaborergebnisse können normal oder sogar erhöht („verbessert“) sein. Autoantikörper gibt es selten. Die zugrunde liegende Ätiologie des avWD ist sehr heterogen und die jeweils zugrunde liegenden Erkrankungen benötigten eine spezielle Behandlung. Behandlungsziel ist es, eine Balance zwischen Blutungs- und Thromboserisiko finden.

\section{Diagnose}

Die klinischen Zeichen der avWD zeigen eine Störung der primären Hämostase (Epistaxis und andere Schleimhautblutungen, subkutane Einblutungen, Petechien, Sickerblutung an Punktionsstellen). Die spezielle Labordiagnostik ist nicht einfach möglich, da normale oder erhöhte vWF-Werte pathologische Befunde verschleiern. Eine Grundvoraussetzung für Spezialtests der Thrombozytenfunktion (z.B. PFA), insbesondere eine fehlende Entzündungsreaktionen, sind unter intensivmedizinischen Bedingungen nicht erfüllt.

\section{Therapie}

Bei der Vielfalt der zugrunde liegenden Ursachen ist die effektive Behandlung der Grunderkrankung von wesentlicher Bedeutung. Zur Gerinnungs- und Hämotherapie verwendet man Antifibrinolytika sowie eine Substitution von Erythrozyten, Thrombozyten und Gerinnungsfaktoren unter klinischer Beobachtung und laborchemischer Überwachung. Die Substitution von vWF bleibt klinischen Studien vorbehalten, solange der vermehrte pathologische Abbau nicht gleichzeitig behandelt werden kann. Im Einzelfall steht für eine gezielte Substitution ein vWF-Monokonzentrat zur Verfügung.

\section{Thrombotische Mikroangiopathien}

Thrombotische Mikroangiopathien treten in Form des hämolytisch-urämischen-Syndroms (HUS) oder der thrombotisch-thrombopenen Purpura (TTP) auf [42]. - Abb. 10 gibt einen Überblick über die Einteilung und Pathogenese. In der weiteren Darstellung hier werden wir uns auf die erworbenen Formen des infektassoziierten HUS und der TTP konzentrieren.

\section{INFOBOX}

Pathogenese des erworbenen von-Willebrand-JürgensSyndroms (avWD)

gesteigerte Clearance des vWF:

- abnorme Bindung an spezifische Antikörper gegen vWF

- Paraproteine (MGUS)

- Medikamente/Kolloide

- Blutzellen

- Endothelzellen

- Tumorzellen

gesteigerte Degradation des vWF:

- arterielle Stenosen

- Gefäßfehlbildungen

- mechanische Herzklappen

- extrakorporale Zirkulationssysteme

- Hyperfibrinolyse

- Graft-versus-Host Disease (GvH)

erniedrigte Synthese/Freisetzung von vWF:

- Hypothyreose

- Urämie

- Medikamente (z. B. Valproinsäure)

\section{Pathogenese, Klinik und Diagnose}

Der wesentliche Pathomechanismus aller Formen des HUS ist eine überschießende Komplementaktivierung durch den „alternative pathway“ aufgrund einer Inaktivität des wesentlichen Regulationsfaktors $\mathrm{H}$. Beim infektasozziierten HUS kommt der direkte zytotoxische Schaden durch die von EHEC in die Blutbahn freigesetzten Shiga-Toxine hinzu. Das HUS führt häufig zu schweren renalen Folgeschäden wie einer chronischen Niereninsuffizienz und der Entwicklung einer chronischen Hypertonie.

Der Pathomechanismus der TTP betrifft den Stoffwechsel des vWF, der als große, multimere Einheiten („ultra large vWF“, UL-vWF) in die Zirkulation abgegeben und dort von einer Metalloproteinase (ADAMTS13) gespalten wird. Bei einem Mangel an dieser Metalloproteinase (bei der erworbenen TTP durch IgG-Autoantikörper) kumuliert UL-vWF. Gerade UL-vWF haben ein hohes thrombogenes Potenzial. Neueste Erkenntnisse legen einen noch engeren Zusammenhang zwischen HUS und TTP als bislang angenommen nahe, da vWF die Komplementantwort modulieren kann. Allerdings kann der Nachweis von UL-vWF nicht als Diagnosekriterium der TTP herangezogen werden, da UL-vWF gerade im akuten Schub in den Thromben gebunden und daher im Blut nicht nachweisbar ist.

Bei beiden Formen der TMA kommt es zu disseminierten thrombotischen Verschlüssen der Mikrostrombahn mit konsekutiver Ischämie und zusätzlicher erythrozy- 


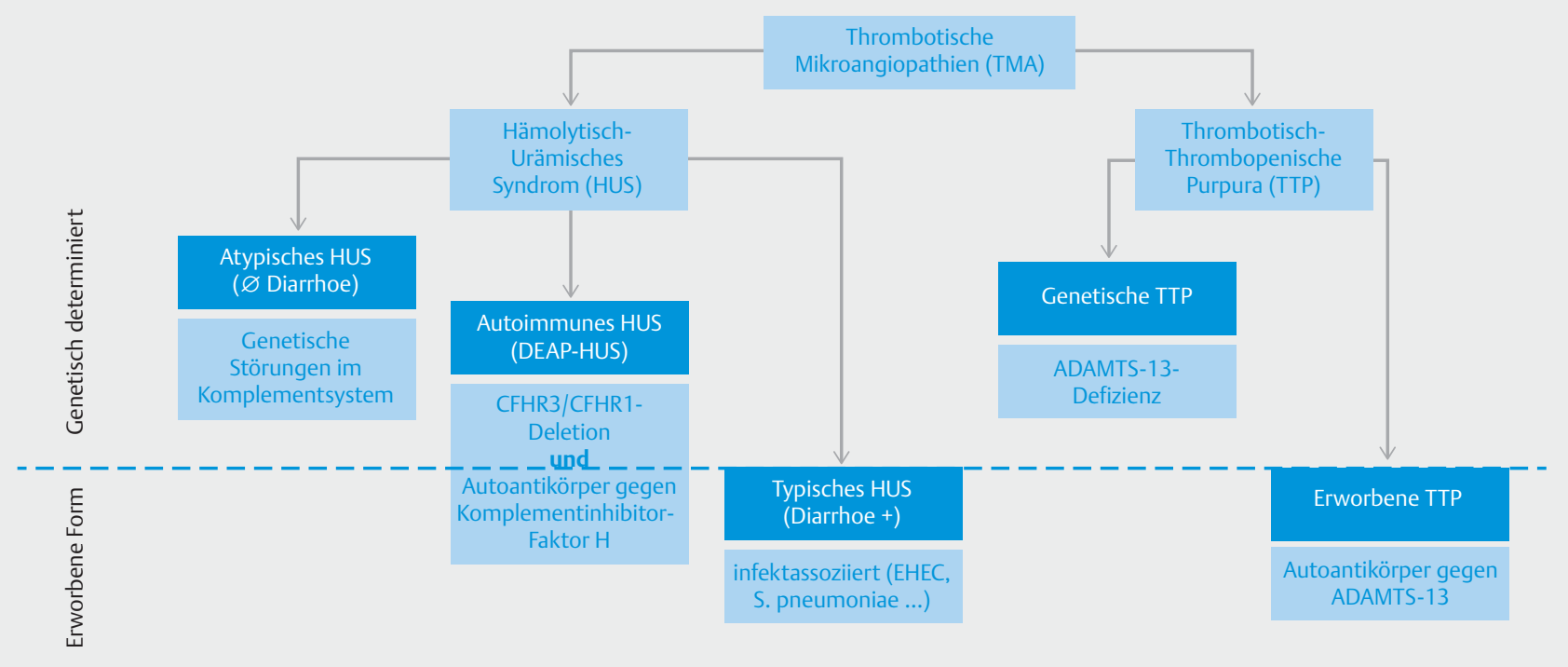

- Abb. 10 Einteilung der thrombotischen Mikroangiopathien und ihre Pathogenese (ADAMTS-13: vWF-spaltende Metalloproteinase; DEAPHUS: „deficient for CFHR proteins and autoantibodies positive for the complement inhibitor factor H“; EHEC: enterohämolytische Escherichia coli).

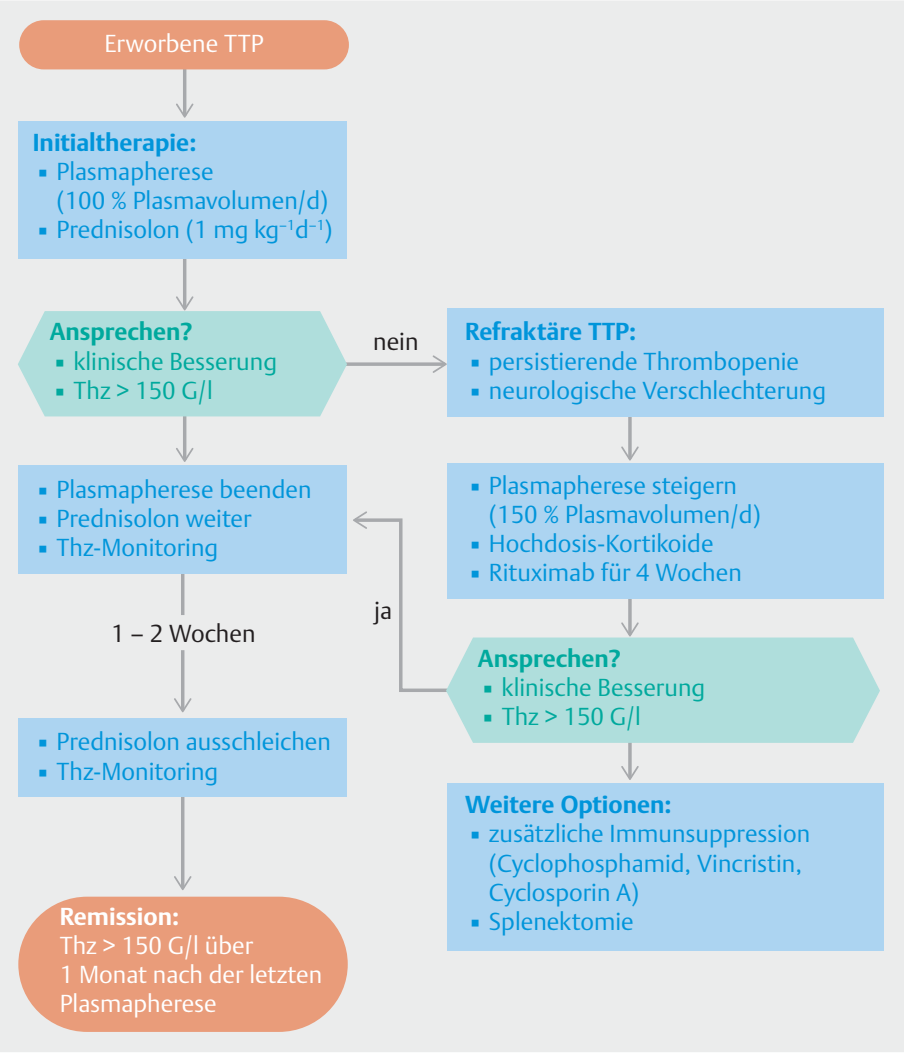

Abb. 11 Therapie der erworbenen thrombotisch-thrombopenen Purpura [44]. tärer Hämolyse. Bei der Klinik des HUS stehen typischerweise akute und im Verlauf chronische Nierenschäden im Vordergrund, während bei der TTP neben den namensgebenden kutanen Einblutungen zentralnervöse Symptome typisch sind.

Eine Gerinnungshemmung oder antithrombozytäre Therapie ist nach den vorliegenden Daten nicht indiziert [43].

\section{Therapie des hämolytisch-urämischen-Syndroms}

Die Therapie des infektassoziierten HUS ist nur unzureichend evidenzbasiert. Wesentliche Erkenntnisse stammen aus großen Ausbrüchen 2011 in Deutschland und 1996 in Japan. Aufgrund der zentralen Stellung der deletären Komplementwirkung werden mehrere Strategien zur Begrenzung der Komplementwirkung eingesetzt, insbesondere die Plasmapherese (mit unzureichenden klinischen Ergebnissen), die Immun-Hämoabsorption sowie seit 2010 vor allem der Einsatz von Eculizumab, einem Komplement-Inhibitor mit vergleichsweise guten Ergebnissen.

\section{Antibiose bei EHEC?}

Eine interessante Kontroverse bezieht sich auf die Antibiotikatherapie bei Infektionen mit EHEC, da die Gefahr besteht, dass hierdurch verstärkt Shiga-Toxin in den Blutstrom freigesetzt wird. Daher sollte man vor allem während der Krankheitsphase mit Diarrhoe möglichst rein unterstützend ohne Antibiotikaeinsatz behandeln. Organkomplikationen können früh oder im weiteren Verlauf allerdings den Einsatz von Antibiotika unumgänglich erscheinen lassen; hierzu besteht derzeit sei- 
tens der Deutschen Gesellschaft für Nephrologie die Empfehlung zum Einsatz von Azitromycin mit guten Ergebnissen einer Eradikation. Bei der Epidemie 1996 wurde Fosfomycin erfolgreich eingesetzt. Allgemein gelten folgende Antibiotika(-gruppen) als sicher hinsichtlich einer verstärkten Freisetzung von Shiga-Toxin: Fosfomycin, Aminoglykoside, Carbapeneme, Cephalosporine.

\section{Plasmapherese bei thrombotisch-thrombopener Purpura}

Kern der Therapie der TTP ist der Ersatz von ADAMTS13. Da diese Metalloproteinase nicht als Konzentrat verfügbar ist, kann sie nur durch die Transfusion von Plasma (FFP) zugeführt werden. Um eine ausreichende Aktivität zu erreichen, muss täglich das gesamte Plasmavolumen ersetzt werden, sodass dies nur in Form einer unspezifischen Plasmapherese geschehen kann. Daneben ist eine ggf. eskalierende immunsuppressive Therapie erforderlich, um den gegen ADAMTS-13 gerichteten IgG-Antikörper zu supprimieren. - Abb.11 zeigt ein aktuelles Therapieschema.

\section{Interessenkonflikt}

L. Ney gibt an, dass kein Interessenkonflikt besteht. M. Spannagl hat Vortragshonorare und/oder Forschungsförderung der Firmen CSL Behring, Bayer, Baxter, NovoNordisc, Boehringer Ingelheim, Octapharma, Pfizer und Biotest erhalten.

Über die Autoren

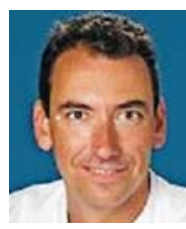

\section{Ludwig Ney}

Dr. med., seit 1995 Mitarbeiter der Klinik für Anaesthesiologie des Klinikums der Universität München. Nach Anerkennung als Facharzt 2000 und der Weiterbildung in der speziellen anästhesiologischen Intensivmedizin 2002 ist er als Oberarzt tätig. Seit 10 Jahren leitet er am Standort Innenstadt die operative Intensivstation an der traditionsreichen Chirurgischen Universitätsklinik in der Nußbaumstraße. Neben der Gerinnungstherapie gilt sein besonderes Interesse der Ernährung kritisch kranker Patienten, der pulmonalen Kreislaufphysiologie sowie der Bedeutung von human factors in der Akutmedizin. Er ist zudem Gründungsmitglied des Ethikkomitees des Universitätsklinikums München.

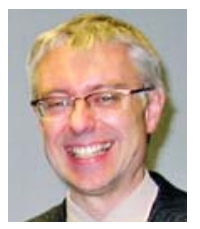

\section{Michael Spannagl}

Prof. Dr. med., Internist, Intensivmediziner, Hämostaseologe und Angiologe. Stv. Leiter der Abteilung für Transfusionsmedizin, Zelltherapeutika und Hämostaseologie, Klinikum der Universität München. Im Vorstand der Gesellschaft für Thrombose- und Hämostaseforschung (GTH e.V.) sowie der Gesellschaft zur Förderung der Qualitätssicherung in medizinischen Laboratorien

\section{KERNAUSSAGEN}

- Häufige Ursachen einer Gerinnungsstörung im klinischen Alltag sind die Antikoagulation und die Thrombozytenhemmung. Ebenfalls nicht selten ist sie Folge einer Erkrankung, u. a. Leber- und Nierenerkrankungen, hämatologische Erkrankungen oder ein Schock.

- Die „Lethal Triade“ (Hypothermie, Azidose, Koagulopathie) führt zu einer Gerinnungsstörung, die chirurgische Maßnahmen, eine hämostaseologische Substitution und eine kardiozirkulatorische Stabilisierung erfordert.

- Das Auftreten diffuser Blutungen ist das wichtigste Leitsymptom einer akuten Koagulopathie.

- Quick und PTT sind schnell und überall verfügbare Globaltests der Gerinnung. Wegen der unterschiedlichen Empfindlichkeit für direkte orale Antikoagulanzien (DOAK) büßen diese Globaltests eine breite diagnostische Aussagekraft aber zusehends ein.

- Therapie der Wahl bei akuter, massiver Blutung ist neben Massivtransfusionen eine frühzeitig Substitution von Fibrinogen und Thrombozyten.

- Klinisch eindeutige Zeichen einer DIC bei Sepsis sind Hautnekrosen und digitale Nekrosen an Händen und Füßen, seltener auch von Ohren, Nase und Mamillen. Der Kern der Therapie einer septischen DIC ist die Antikoagulation mit Heparin (UFH oder NMH).

- Vor allem Autoantikörper gegen die Faktoren des intrinsischen Systems - am häufigsten Faktor VIII (HemmkörperHämophilie), seltener auch gegen Faktor IX und XI - können zu schweren Blutungskomplikationen führen. Diagnostik und Therapie dieser erworbenen Blutungsneigung sind jedoch außerordentlich aufwendig.

(INSTAND e.V.). Mitglied in den Leitlinienkommissionen „Hämotherapie“ und „medizinische Laboratoriumsdiagnostik“ der Bundesärztekammer und der in vitro Diagnostika Arbeitsgruppen bei DIN, CEN und ISO.

\section{Korrespondenzadresse}

Dr. Ludwig Ney

Klinikum der Universität München

Klinik für Anaesthesiologie

Nußbaumstraße 1

80336 München

E-Mail: ludwig.ney@med.uni-muenchen.de 
Literatur

[1] Hoffman M, Monroe DM 3rd. A cell-based model of hemostasis. Thromb Haemost 2001; 85: 958-965

[2] Krumrei N], Park MS, Cotton BA et al. Comparison of massive blood transfusion predictive models in the rural setting. Journal of Trauma and Acute Care Surgery 2012; 72: 211 215

[3] Nunez TC, Voskresensky IV, Dossett LA et al. Early prediction of massive transfusion in trauma: simple as $A B C$ (assessment of blood consumption)? Journal of Trauma 2009; 66: $346-352$

[4] Yucel N, Lefering R, Maegele M et al. Trauma Associated Severe Hemorrhage (TASH)-Score: probability of mass transfusion as surrogate for life threatening hemorrhage after multiple trauma. Journal of Trauma 2006; 60: 1228-1236 ; discussion 1236-1227

[5] McLaughlin DF, Niles SE, Salinas J et al. A predictive model for massive transfusion in combat casualty patients. Journal of Trauma 2008; 64: S57-63; d63

[6] Blatchford O, Murray WR, Blatchford M. A risk score to predict need for treatment for upper-gastrointestinal haemorrhage. Lancet 2000; 356: $1318-1321$

[7] Subherwal S, Bach RG, Chen AY et al. Baseline risk of major bleeding in non-ST-segment-elevation myocardial infarction: the CRUSADE (Can Rapid risk stratification of Unstable angina patients Suppress ADverse outcomes with Early implementation of the ACC/AHA Guidelines) Bleeding Score. Circulation 2009; 119: $1873-1882$

[8] Levine GN, Bates ER, Bittl JA et al. 2016 ACC/AHA Guideline Focused Update on Duration of Dual Antiplatelet Therapy in Patients With Coronary Artery Disease. Circulation 2016: DOI 10.1161/CIR.0000000000000404

[9] Kirchhof P, Benussi S, Kotecha D et al. 2016 ESC Guidelines for the management of atrial fibrillation developed in collaboration with EACTS. Eur Heart J 2016: DOI 10.1093/eurheartj/ehw210

[10] Pisters R, Lane DA, Nieuwlaat $R$ et al. A novel user-friendly score (HAS-BLED) to assess 1-year risk of major bleeding in patients with atrial fibrillation: the Euro Heart Survey. Chest 2010; 138: $1093-1100$

[11] O'Brien EC, Simon DN, Thomas LE et al. The ORBIT bleeding score: a simple bedside score to assess bleeding risk in atrial fibrillation. Eur Heart J 2015: DOI 10.1093/eurheartj/ ehv476

[12] Hijazi Z, Oldgren J, Lindback J et al. The novel biomarkerbased ABC (age, biomarkers, clinical history)-bleeding risk score for patients with atrial fibrillation: a derivation and validation study. Lancet 2016; 387: 2302-2311

[13] Müller MC, Meijers JC, Vroom MB et al. Utility of thromboelastography and/or thromboelastometry in adults with sepsis: a systematic review. Crit Care 2014; 18: R30

[14] Young T, Tang H, Hughes R. Vena caval filters for the prevention of pulmonary embolism. Im Internet: http://onlinelibrary.wiley.com/doi/10.1002/14651858.CD006212. pub4/abstract

[15] Douketis JD, Spyropoulos AC, Spencer FA et al. Perioperative management of antithrombotic therapy: Antithrombotic therapy and prevention of thrombosis, 9th ed: american college of chest physicians evidence-based clinical practice guidelines. Chest 2012; 141: e326S-e350S
[16] Korte W, Cattaneo M, Chassot PG et al. Peri-operative management of antiplatelet therapy in patients with coronary artery disease. Thromb Haemost 2011; 105: 743 - 749

[17] Falck-Ytter Y, Francis CW, Johanson NA et al. Prevention of VTE in orthopedic surgery patients: Antithrombotic therapy and prevention of thrombosis: american college of chest physicians evidence-based clinical practice guidelines. Chest 2012; 141: e278S-e325S

[18] Kahn SR, Lim W, Dunn AS et al. Prevention of VTE in nonsurgical patients: Antithrombotic therapy and prevention of thrombosis, 9th ed: american college of chest physicians evidence-based clinical practice guidelines. Chest 2012; 141: e195S-e226S

[19] Charbit B, Mandelbrot L, Samain E et al. The decrease of fibrinogen is an early predictor of the severity of postpartum hemorrhage. J Thromb Haemost 2007; 5: 266 - 273

[20] Schlembach D, Mortl MG, Girard T et al. Management of postpartum hemorrhage (PPH): algorithm of the interdisciplinary D-A-CH consensus group PPH (Germany - Austria Switzerland). Anaesthesist 2014; 63: 234-242

[21] Kozek-Langenecker S, Sorensen B, Hess JR et al. Clinical effectiveness of fresh frozen plasma compared with fibrinogen concentrate: a systematic review. Crit Care 2011; 15 : R239

[22] Estcourt LJ, Stanworth S], Doree C et al. Comparison of different platelet count thresholds to guide administration of prophylactic platelet transfusion for preventing bleeding in people with haematological disorders after myelosuppressive chemotherapy or stem cell transplantation. Cochrane Database Syst Rev 2015; 11: CD010983

[23] Maegele M, Schochl H, Cohen M]. An update on the coagulopathy of trauma. Shock 2014; 41 (Suppl. 01): $21-25$

[24] Davenport RA, Brohi K. Coagulopathy in trauma patients: importance of thrombocyte function? Curr Opin Anaesthesiol 2009; 22: $261-266$

[25] Besser MW, Ortmann E, Klein AA. Haemostatic management of cardiac surgical haemorrhage. Anesthesia 2015; 70 (Suppl. 01): $87-95$

[26] Thachil J. Disseminated Intravascular Coagulation: A Practical Approach. Anesthesiology 2016: DOI 10.1097/ ALN.0000000000001123

[27] Shakur H, Roberts I, Bautista R et al. Effects of tranexamic acid on death, vascular occlusive events, and blood transfusion in trauma patients with significant haemorrhage (CRASH-2): a randomised, placebo-controlled trial. Lancet 2010; 376: $23-32$

[28] Spahn DR, Bouillon B, Cerny V et al. Management of bleeding and coagulopathy following major trauma: an updated European guideline. Crit Care 2013; 17: R76

[29] Moore HB, Moore EE, Liras IN et al. Acute Fibrinolysis Shutdown after Injury Occurs Frequently and Increases Mortality: A Multicenter Evaluation of 2,540 Severely Injured Patients. J Am Coll Surg 2016; 222: 347 - 355

[30] Warren BL, Eid A, Singer P et al. Caring for the critically ill patient. High-dose antithrombin III in severe sepsis: a randomized controlled trial. JAMA 2001; 286: 1869-1878

[31] Gando S, Saitoh D, Ishikura H et al. A randomized, controlled, multicenter trial of the effects of antithrombin on disseminated intravascular coagulation in patients with sepsis. Crit Care 2013; 17: R297

[32] Eichacker PQ, Natanson C, Danner RL. Surviving sepsis practice guidelines, marketing campaigns, and Eli Lilly. $\mathrm{N}$ Engl J Med 2006; 355: 1640 - 1642 
[33] Marti-Carvajal A], Sola I, Gluud C et al. Human recombinant protein $\mathrm{C}$ for severe sepsis and septic shock in adult and paediatric patients. Cochrane Database Syst Rev 2012; 12 : CD004388

[34] Yamakawa K, Aihara M, Ogura H et al. Recombinant human soluble thrombomodulin in severe sepsis: a systematic review and meta-analysis. J Thromb Haemost 2015; 13: 508 519

[35] Dickneite G, Herwald H, Korte W et al. Coagulation factor XIII: a multifunctional transglutaminase with clinical potential in a range of conditions. Thromb Haemost 2015; 113: 686-697

[36] Weber CF, Sanders JO, Friedrich K et al. Stellenwert der Thrombelastometrie für das Monitoring von Faktor XIII. Prospektive Observationsstudie bei neurochirurgischen Patienten. Hämostaseologie 2011; 31: 111-117

[37] Wettstein P, Haeberli A, Stutz M et al. Decreased factor XIII availability for thrombin and early loss of clot firmness in patients with unexplained intraoperative bleeding. Anesth Analg 2004; 99: $1564-1569$

[38] Godje O, Gallmeier U, Schelian M et al. Coagulation factor XIII reduces postoperative bleeding after coronary surgery with extracorporeal circulation. Thorac Cardiovasc Surg 2006; 54: $26-33$

[39] Karkouti K, von Heymann C, Jespersen CM et al. Efficacy and safety of recombinant factor XIII on reducing blood transfusions in cardiac surgery: a randomized, placebo-controlled, multicenter clinical trial. J Thorac Cardiovasc Surg 2013; 146: $927-939$
[40] Tengborn L, Baudo F, Huth-Kuhne A et al. Pregnancy-associated acquired haemophilia $A$ : results from the European Acquired Haemophilia (EACH2) registry. BJOG 2012; 119: $1529-1537$

[41] Stemberger M, Mohnle P, Tschop J et al. Successful bleeding control with recombinant porcine factor VIII in reduced loading doses in two patients with acquired haemophilia A and failure of bypassing agent therapy. Haemophilia 2016; 22: e472-474

[42] Zipfel PF, Wolf G, John U et al. Novel developments in thrombotic microangiopathies: is there a common link between hemolytic uremic syndrome and thrombotic thrombocytic purpura? Pediatr Nephrol 2011; 26: 1947 1956

[43] Wurzner R, Riedl M, Rosales A et al. Treatment of enterohemorrhagic Escherichia coli-induced hemolytic uremic syndrome (eHUS). Semin Thromb Hemost 2014; 40: 508 516

[44] Sayani FA, Abrams CS. How I treat refractory thrombotic thrombocytopenic purpura. Blood 2015; 125: $3860-3867$

Bibliografie

DOI http://dx.doi.org/10.1055/s-0042-111029 Intensivmedizin up2date 2017; 13: 27-47

(C) Georg Thieme Verlag KG Stuttgart · New York ISSN 1614-4856 


\section{Punkte sammeln auf CME.thieme.de}

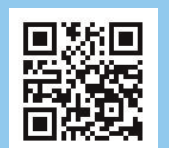

Diese Fortbildungseinheit ist 12 Monate online für die Teilnahme verfügbar.

Sollten Sie Fragen zur Online-Teilnahme haben, finden Sie unter http://cme.thieme.de/hilfe eine ausführliche Anleitung. Wir wünschen viel Erfolg beim Beantworten der Fragen!

Unter https://eref.thieme.de/ZZWHE7N oder über den QR-Code kommen Sie direkt zum Artikel zur Eingabe der Antworten.

VNR 2760512017152372118

\section{Frage 1}

Welcher Befund spricht für sich am wenigsten für eine akute Koagulopathie beim Intensivpatienten?

A Blutung aus Stichkanälen und Wunden

B Blutung beim Absaugen

C spontane Hämatome

D Blutung aus Ösophagusvarizen

E Schleimhautblutungen

\section{Frage 2}

Bei welcher Erkrankung führt man die medikamentöse Rezidivprophylaxe nicht mit Gerinnungshemmstoffen durch?
A Vorhofflimmern
B Venenthrombose
C koronare Herzerkrankung
D Sinusvenenthrombose
E zerebrale Ischämie bei Kunstklappe

\section{Frage 3}

Wobei besteht kein erhöhtes Blutungsrisiko unter Antikoagulation?

A nach gastrointestinaler Blutung

B gleichzeitige Einnahme von Azetylsalizylsäure

C vorbekannte Lebererkrankung

D vorbekannte Nierenerkrankung

E vorbekannte chronisch obstruktive Lungenerkrankung

\section{Frage 4}

Worauf weist eine plötzlich auftretende isolierte PTT-Verlängerung mit deutlichen klinischen Blutungszeichen beim Patienten hin?
A Immunthrombopenie
B erworbene Hemmkörper-Hämophilie
C parenterale Antikoagulation
D Lupus-Antikoagulanzien
E Faktor-XII-Mangel

\section{Frage 5}

Welche Aussage zur Behandlung der akuten Koagulopathie nach Trauma ist richtig?

A Die Gabe von Fibrinogenkonzentrat potenziert die Thrombosegefahr.

B Die Gabe von fibrinstabilisierendem Faktor (Faktor XIII) ist die Therapie der Wahl der Hyperfibrinolyse nach Trauma.

C Beim erwachsenen Patienten (ca. $70 \mathrm{kgKG}$ ) führt bereits die Transfusion von $250 \mathrm{ml}$ Frischplasma zu einem meist ausreichenden Anstieg der Gerinnungsaktivität.

D Bei der Massivtransfusion sollte das Verhältnis zwischen Erythrozytenkonzentraten und FFP 1:1 bis maximal 2:1 betragen.

E Die Transfusion von Thrombozyten bei akuter Blutung ist aufgrund der traumainduzierten Thrombopathie wirkungslos.

\section{Frage 6}

Welche Aussage zur Gerinnungstherapie bei septischen Patienten ist richtig?

A In der Sepsis führt die disseminierte intravasale Gerinnung zur einer Verbrauchskoagulopathie, die häufig mit einer massiven Hyperfibrinolyse einhergeht.

B Der Einsatz von Thrombinantagonisten zur Antikoagulation ist bei septischen Patienten streng kontraindiziert.

C Sowohl bei einer traumatischen Blutung als auch in der Sepsis führt eine überschießende Aktivierung des extrinsischen Gerinnungswegs zu einer Verbrauchskoagulopathie. Daher sollte man auch in der Sepsis vorsorglich Antifibrinolytika einsetzen (z. B. Tranexamsäure).

D In der Sepsis sollte man frühzeitig mit einer Thromboseprophylaxe (low dose Heparinisierung i. v. oder niedermolekulares Heparin s.c.) beginnen.

E Eine hoch- oder supranormale Antithrombinaktivität ( $\geq 110 \%$ ) führt zu einer signifikanten Outcome-Verbesserung bei septischen Patienten. 


\title{
CME-Fragen bei CME.thieme.de
}

\author{
Fortsetzung ...
}

\section{Frage 7}

Welche Aussage zur Hemmkörper-Hämophilie ist richtig?

A Eine Hemmkörper-Hämophilie tritt überwiegend bei Hämophilie-Patienten unter langjähriger Substitutionstherapie auf, da sich Antikörper gegen den allogenen Faktor VIII bilden.

B Bei der Hemmkörper-Hämophilie sollte man bei einer akuten Blutung frühzeitig die Transfusion von FFP erwägen.

C Die typische Laborkonstellation bei der HemmkörperHämophilie ist die gleichzeitige Verlängerung der PTT mit einem starken Abfall des Quick-Werts.

D Die Hemmkörper-Hämophilie geht in über zwei Dritteln der Fälle auf eine vorbestehende Autoimmunerkrankung wie Rheuma oder systemischer Lupus erythematodes zurück.

E Zur Therapie der Hemmkörper-Hämophilie gehört auch eine oft längere Zeit erforderliche Immunsuppression.

\section{Frage 8}

Das erworbene von-Willebrand-Jürgens-Syndrom hat vielfältige Ursachen. Welche Erkrankung gehört nicht dazu?

A myeloproliferative Erkrankungen

B stenosierende Herzklappenerkrankungen

C LVAD („left ventricular assist device“)

D Paraproteinämie

E Autoimmunthrombozytopenie

\section{Frage 9}

Bei Patienten mit Thrombozytopenie muss man die verursachende Störung berücksichtigen. In welcher Situation ist trotz der erniedrigten Zellzahl eine therapeutische Antikoagulation erforderlich?

A Thrombozytopenie bei Multitransfusion

B heparininduzierte Thrombozytopenie Typ II

C Autoiimunthrombozytopenie (Morbus Werlhof)

D Thrombozytopenie unter Chemotherapie

E Pseudothrombozytopenie

\section{Frage 10}

Welche Aussage zur Diagnostik von Gerinnungsstörungen ist falsch?

A Die publizierten Scoringsysteme erlauben keine zuverlässige individualisierte Vorhersage von Blutungskomplikationen unter Antikoagulation.

B Neben der unfall- bzw. schockbedingten Hypothermie und Azidose können auch iatrogene Faktoren, insbesondere die Infusions- und Transfusionstherapie zu einer Verstärkung einer traumabedingten Koagulopathie beitragen.

C Bereits in der akuten Blutungssituation sollte man mit einer Antikoagulation beginnen, um der Entwicklung einer Verbrauchskoagulopathie zu begegnen.

D Die Aussagekraft der thrombozytären In-vitro-Funktionsdiagnostik (z. B. PFA 100, Multiplate) ist bei Thrombozytopenie und Anämie eingeschränkt.

E Eine therapeutische Antikoagulation oder Thrombozytenfunktionshemmung ist im klinischen Alltag die häufigste Ursache von Gerinnungsstörungen. 